\title{
Mapping of Genomic Vulnerabilities in the Post-Translational Ubiquitination, SUMOylation and Neddylation Machinery in Breast Cancer
}

\author{
Jesús Fuentes-Antrás ${ }^{1, *}+{ }^{\dagger}$, Ana Lucía Alcaraz-Sanabria ${ }^{2,+}$, Esther Cabañas Morafraile ${ }^{1}$, \\ María del Mar Noblejas-López ${ }^{2} \mathbb{D}$, Eva María Galán-Moya ${ }^{2} \mathbb{D}$, Mariona Baliu-Pique ${ }^{1}$, Igor López-Cade ${ }^{3} \mathbb{D}$, \\ Vanesa García-Barberán ${ }^{3}$ (D) Pedro Pérez-Segura ${ }^{1}$, Aránzazu Manzano ${ }^{1}$, Atanasio Pandiella ${ }^{4}$, Balázs Győrffy ${ }^{5,6,7}$ \\ and Alberto Ocaña ${ }^{1,2, *}$
}

check for updates

Citation: Fuentes-Antrás, J.; Alcaraz-Sanabria, A.L.; Morafraile, E.C.; Noblejas-López, M.d.M.; Galán-Moya, E.M.; Baliu-Pique, M.; López-Cade, I.; García-Barberán, V.; Pérez-Segura, P.; Manzano, A.; et al. Mapping of Genomic Vulnerabilities in the Post-Translational Ubiquitination, SUMOylation and Neddylation Machinery in Breast Cancer. Cancers 2021, 13, 833. https://doi.org/10.3390/cancers 13040833

Academic Editor: Tarek Abbas

Received: 8 January 2021

Accepted: 14 February 2021

Published: 17 February 2021

Publisher's Note: MDPI stays neutral with regard to jurisdictional claims in published maps and institutional affiliations.

Copyright: (C) 2021 by the authors. Licensee MDPI, Basel, Switzerland. This article is an open access article distributed under the terms and conditions of the Creative Commons Attribution (CC BY) license (https:/ / creativecommons.org/licenses/by/ $4.0 /)$.
1 Experimental Therapeutics Unit, Hospital Clínico Universitario San Carlos (HCSC), Instituto de Investigación Sanitaria San Carlos (IdISSC) and Centro de Investigación Biomédica en Red en Oncología (CIBERONC), 28040 Madrid, Spain; esther.cabanas@salud.madrid.org (E.C.M.); maria.baliu.pique@sergas.es (M.B.-P.); pedro.perez@salud.madrid.org (P.P.-S.); aranzazu.manzano@salud.madrid.org (A.M.)

2 Translational Oncology Laboratory, Centro Regional de Investigaciones Biomédicas, Castilla-La Mancha University (CRIB-UCLM), 02008 Albacete, Spain; analucia.alcaraz@uclm.es (A.L.A.-S.); mariadelmar.noblejas@uclm.es (M.d.M.N.-L.); evamaria.galan@uclm.es (E.M.G.-M.)

3 Molecular Oncology Laboratory, Instituto de Investigación Sanitaria San Carlos (IdISCC), 28040 Madrid, Spain; ilopez.7@alumni.unav.es (I.L.-C.); vanesa.garciabar@salud.madrid.org (V.G.-B.)

4 Instituto de Biología Molecular y Celular del Cáncer (IBMCC), Consejo Superior de Investigaciones Científicas (CSIC-IBSAL) and Centro de Investigación Biomédica en Red en Oncología (CIBERONC), 37007 Salamanca, Spain; atanasio@usal.es

5 Department of Bioinformatics, Semmelweis University, H-1094 Budapest, Hungary; gyorffy.balazs@ttk.hu 6 2nd Department of Pediatrics, Semmelweis University, H-1094 Budapest, Hungary

7 TTK Cancer Biomarker Research Group, Institute of Enzymology, H-1117 Budapest, Hungary

* Correspondence: jfuentesa@salud.madrid.org (J.F.-A.); alberto.ocana@salud.madrid.org (A.O.)

+ These authors contributed equally to this paper.

Simple Summary: Breast cancer is a major cause of death worldwide and remains incurable in advanced stages. The dysregulation of the post-translational machinery has been found to underlie tumorigenesis and drug resistance in preclinical models but has only recently led to early trials in cancer patients. We performed an in silico analysis of the most common genomic alterations occurring in ubiquitination and ubiquitin-like SUMOylation and neddylation using data from publicly available repositories and with the aim of identifying those with prognostic and predictive value and those exploitable for therapeutic intervention. Clinical and statistical criteria were used to sort out the best candidates and the results were validated in independent datasets. UBE2T, UBE2C, and BIRC5 amplifications predicted a worse survival and poor response to therapy across different intrinsic subtypes of breast cancer. Mutated USP9X and USP7 also conferred detrimental outcome. Leveraging these molecular vulnerabilities as biomarkers or drug targets could benefit breast cancer patients.

Abstract: The dysregulation of post-translational modifications (PTM) transversally impacts cancer hallmarks and constitutes an appealing vulnerability for drug development. In breast cancer there is growing preclinical evidence of the role of ubiquitin and ubiquitin-like SUMO and Nedd8 peptide conjugation to the proteome in tumorigenesis and drug resistance, particularly through their interplay with estrogen receptor signaling and DNA repair. Herein we explored genomic alterations in these processes using RNA-seq and mutation data from TCGA and METABRIC datasets, and analyzed them using a bioinformatic pipeline in search of those with prognostic and predictive capability which could qualify as subjects of drug research. Amplification of $U B E 2 T, U B E 2 C$, and BIRC5 conferred a worse prognosis in luminal A/B and basal-like tumors, luminal A/B tumors, and luminal A tumors, respectively. Higher $U B E 2 T$ expression levels were predictive of a lower rate of pathological complete response in triple negative breast cancer patients following neoadjuvant chemotherapy, whereas $U B E 2 C$ and BIRC5 expression was higher in luminal A patients with tumor relapse within 5 years of endocrine therapy or chemotherapy. The transcriptomic signatures of USP9X and USP7 gene 
mutations also conferred worse prognosis in luminal A, HER2-enriched, and basal-like tumors, and in luminal A tumors, respectively. In conclusion, we identified and characterized the clinical value of a group of genomic alterations in ubiquitination, SUMOylation, and neddylation enzymes, with potential for drug development in breast cancer.

Keywords: post-translational modification; ubiquitination; SUMOylation; neddylation; breast cancer; biomarkers; prognosis

\section{Introduction}

Despite the continuous improvement of diagnostic and therapeutic strategies, virtually all patients with advanced breast cancer (BC) will die from this disease [1]. In recent years, large-scale genomic studies have generated an overwhelming pool of data that reflects the complexity and heterogeneity of $\mathrm{BC}$ [2-6]. From a clinical standpoint, the progressive genomic characterization has helped envision potential druggable targets based on genomic vulnerabilities, some of which have reached clinical implementation at different levels as exemplified by the growing importance of the ESMO Scale of Clinical Actionability in clinical trial design [7-9]. However, a large number of molecular alterations remain unexplored, including many with potential for medical development.

Posttranslational modifications (PTM), defined as the covalent and generally enzymatic modification of proteins following biosynthesis, are progressively gaining momentum in cancer research as they play a transversal role in many biological processes found aberrant in carcinogenesis, including protein diversification, degradation and recycling, gene regulation, oncogenic signaling (i.e., p53, NF-kB, TGF- $\beta$ pathways) and DNA damage repair $[10,11]$. These modifications encompass phosphorylation, acetylation, glycosylation, and ubiquitination, among many others, and are subject to exquisite regulatory mechanisms often altered in cancer. Considering their critical roles and their widespread dysregulation in cancer, there is a growing interest in developing therapeutic approaches capable of specifically exploiting the ubiquitin and ubiquitin-like pathways (namely, SUMO and Nedd8) in solid tumors. From a molecular perspective, ubiquitin is a small 76-amino-acids protein that can be conjugated to target proteins by three types of enzymes including ubiquitin-activating enzymes (E1s), ubiquitin-conjugating enzymes (E2s), and ubiquitin ligases (E3s) [12]. Small ubiquitin-like modifier (SUMO) and Nedd8 are ubiquitin-like proteins with the same enzymatic structural design as ubiquitin modification, requiring E1-activating, E2-conjugating, and E3-ligating enzymes. Although these three PTMs (SUMOylation, ubiquitination, and neddylation; collectively termed SUN) exert pleiotropic functions in common processes such as cell signaling, inflammatory responses, and DNA damage repair, ubiquitination plays a primary role in the degradation and recycling of the proteome, while SUMOylation and neddylation participate in protein stabilization, transcriptional regulation, epithelial-mesenchymal transformation, and apoptosis [10,11,13-15].

Drug development based on molecular vulnerabilities occurring in the SUN machinery has only recently emerged, although the ubiquitin-conjugation-associated proteasome pathway is already targeted by FDA approved drugs (i.e., Bortezomib) used in malignancies such as multiple myeloma [16]. A comprehensive overview of preclinical and ongoing human trials targeting multiple SUN components was recently published by Gâtel et al., with most research focused on hematologic malignancies and in early phases of development [11]. In BC models, the targeted inhibition of MDM2, an E3-ubiquitin-ligase, has shown promising therapeutic effects either through its autoubiquitination and proteasomal degradation by SP-141 or by blocking the p53-MDM2 interaction by WK298 and SJ-172550 [17,18]. Interestingly, proteolysis targeting chimeras (i.e. PROTACs), developed to trigger ubiquitination and subsequent degradation of specific proteins, have recently proved to exert superior tumor growth inhibition compared to fulvestrant by targeting the 
estrogen receptor (ER) in a patient-derived xenograft model from a patient harboring a mutation in the ER, and a similar PROTAC is currently being tested in ER positive HER2 negative BC patients in a phase I trial (NCT04072952) [19]. Furthermore, these basic mechanisms of proteostasis have not been studied as potential biomarkers of drug efficacy or prognosis in $\mathrm{BC}$.

In this article, we explored genomic alterations in the SUN machinery occurring in BC. With a clinical scope and in search of potential actionability, we focused on amplifications with a prognostic correlation and leading to increased levels of enzymes, thereby filtering for intuitive targets for drug development. By interrogating multiple large publicly accessible databases and analytical webs for cancer omics, we found that gene amplifications are the most frequent genomic alteration of the SUN components. An association with a worse clinical outcome was identified for SUMO2, TCEB2, BIRC5, UBE2T, DERL1, PRKDC, and $U B E 2 C$ gene amplifications. We further described that the amplification of UBE2T, $U B E 2 C$, and $B I R C 5$, remain significant and clinically relevant predictors of a worse outcome in luminal A/B and basal-like, luminal A/B, and luminal A subtypes, respectively. This prognostic signature also showed predictive potential after stratifying by treatments administered in the (neo)adjuvant setting. Finally, although less common, mutations in USP9X and USP7 also predicted a worse outcome in different intrinsic subtypes. In summary, we report the potential prognostic and predictive capability of genomic alterations in SUN enzymes and provide a rationale for future drug development.

\section{Results}

\subsection{Copy Number Alterations in SUMOylation, Ubiquitination, and Neddylation in Breast Cancer}

Reactome, a curated database of biological pathway knowledge, was sequentially interrogated for genes involved in the metabolism of proteins, post-translational protein modifications, and specifically the processes of SUMOylation, ubiquitination, and neddylation. A total of 113 genes were identified (Figure 1a). Of them nine, 77, and 28 genes were involved in SUMOylation, ubiquitination, and neddylation, respectively (Figure 1b). Only one gene (UCHL3) belonged to two groups (neddylation and ubiquitination). In order to limit redundance, we confirmed that such functional characterization was recapitulated in the EnrichR database (Figure 1c). We explored the TCGA (Cell 2015) and METABRIC datasets in the cBioPortal online platform to identify frequent alterations in these genes in BC irrespective of their intrinsic subtype (Figure 1d; Figure S1) [20,21]. Tumor mutational and copy number alteration (CNA) data was available in 2989 of patients combining both datasets. Gene amplifications were by far the most frequent alterations occurring in SUN processes, with the highest proportion observed in the UBE2T gene, in $23.46 \%$ of patients. In this work, we focused on the study of these alterations since genomic amplifications, and subsequently increased levels of enzymes, underlie many oncogenic pathways and resistance mechanisms and thereby provide intuitive targets for drug development. 
(a)

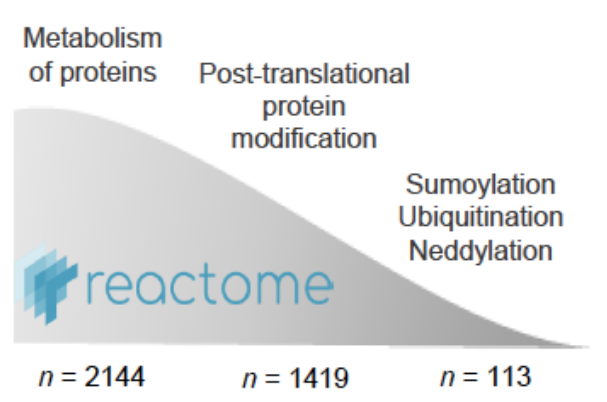

(d)

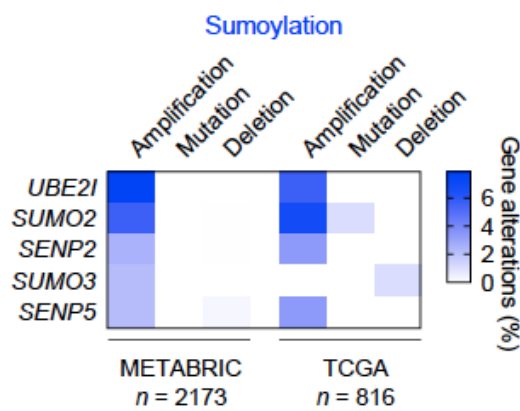

(c)

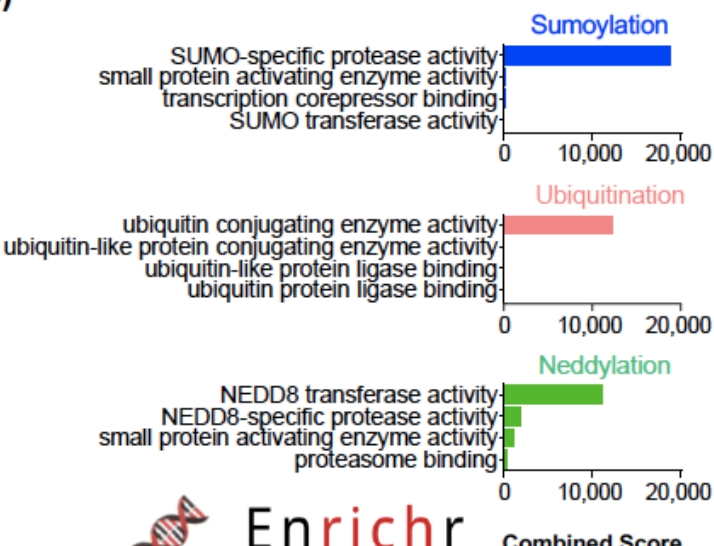

(b)

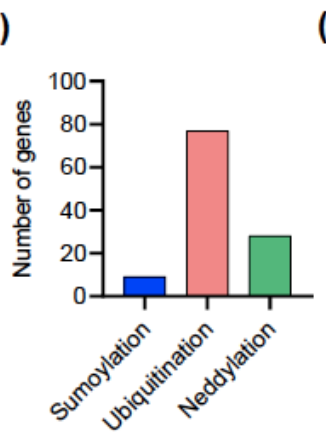

Enrichr

Figure 1. Common genomic alterations in ubiquitination, SUMOylation, and neddylation in breast cancer. (a) Flow chart showing the interrogation of the Reactome database to identify genes involved in post-translational protein modifications, particularly ubiquitination, SUMOylation, and neddylation. (b) Bar graph summarizing the number of genes participating in each process. Only UCHL3 participated in both ubiquitination and neddylation. (c) Bar graph showing the GO molecular function of the genes previously identified for each process, sorted by the combined score in the EnrichR database. (d) Heatmap presenting the frequency of patients from the TCGA and METABRIC datasets with amplifications, mutations, and deletions, of the genes involved in each process. Only genes with a frequency of amplifications higher than $2 \%$ are displayed, those under $2 \%$ can be found in Figure S1.

\subsection{Filtering of Gene Amplifications in Search of Potentially Relevant Clinical Targets}

Next, we explored the concordance of gene amplifications across the two datasets to identify those alterations homogenously distributed in the whole population available. We performed a regression analysis and set a $2 \%$ alteration frequency threshold to curate for potential clinical relevance (Figure 2a). The selected genes were further analyzed according to their amplification frequency across the different intrinsic subtypes of BC, with no evident clustering of amplifications in any of them (Figure 2b). 
(a)
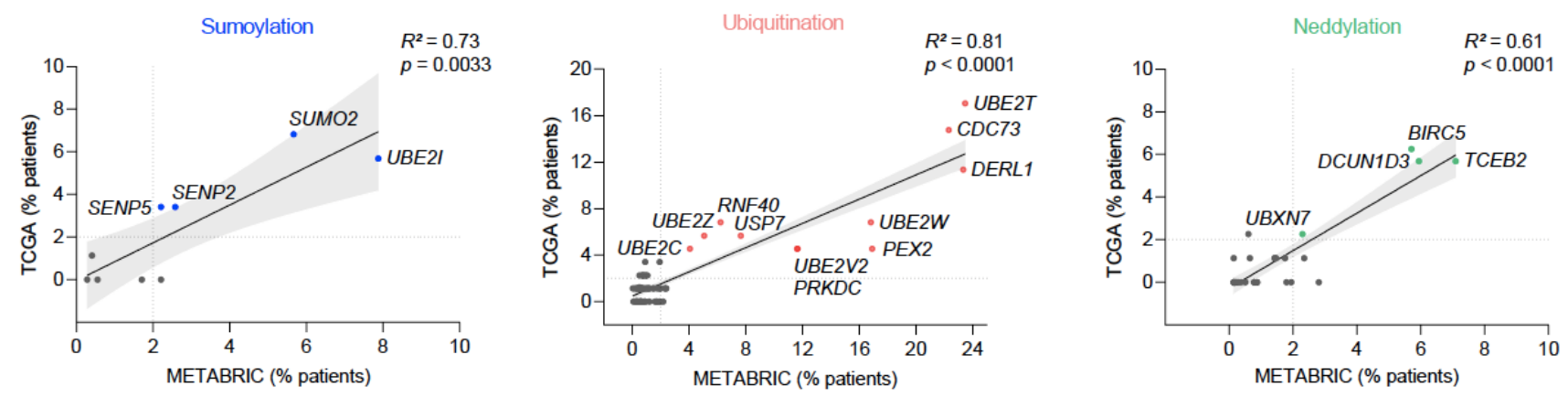

(b)
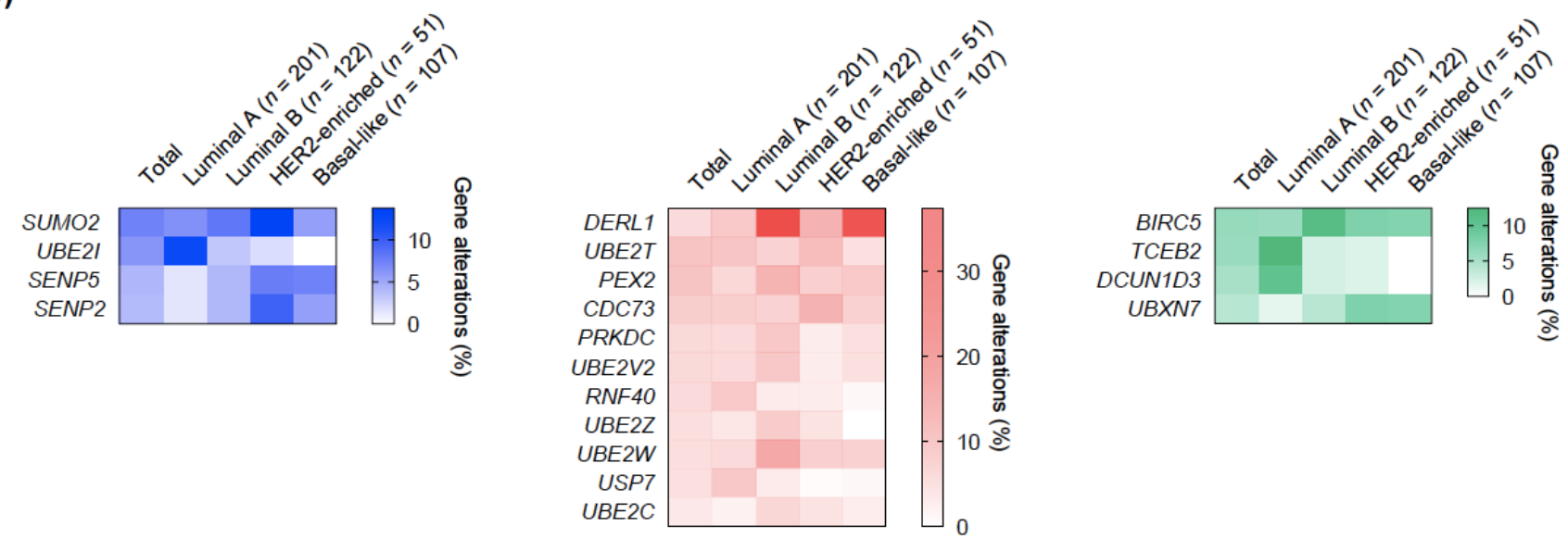

Figure 2. Filtering of gene amplifications. (a) Regression analysis of amplification frequencies in the TCGA and METABRIC datasets, highlighting gene amplifications that occur in more than $2 \%$ of patients in both. (b) Distribution of the frequency of the selected amplified genes across intrinsic subtypes in patients from the TCGA dataset. Population is limited to those patients in which the intrinsic subtype is available in cBioPortal for stratification.

To evaluate whether gene amplifications truly impacted mRNA levels, we first interrogated the GEPIA2 web tool [22,23]. Significantly increased mRNA levels were found in breast tumors compared to healthy controls for UBE2T, UBE2C, BIRC5, TCEB2 (Figure 3). Although non-significant, most of the other genes consistently showed increased mRNA levels in tumor samples. Further, the bc-GenExMiner 3.0 and UALCAN databases were used to study how the expression of those genes with significantly increased mRNA levels distributed across the different intrinsic subtypes and tumor stages [23,24]. Remarkably, mRNA levels of UBE2T, UBE2C, and BIRC5, were found significantly higher in basal-like tumors compared to luminal A tumors, whereas TCEB2 mRNA levels were found higher in luminal A tumors compared to basal-like tumors, although this difference was slight. By analyzing individual patient data from the TCGA database, we confirmed that patients with gene amplifications had higher transcriptional levels across the different intrinsic subtypes (Figure 3). For all genes, expression tended to be highest in stage IV samples and lowest in stage I (Figure S2a). Similarly, protein levels were highest in basal-like tumors and lowest in luminal tumors, with the exception of TCEB2, thus pointing to a positive correlation between the protein burden and a more dedifferentiated phenotype (Figure $\mathrm{S} 2 \mathrm{~b})$. Also of note, a significantly lower promoter methylation level was found in the $U B E 2 T$ gene in $B C$ samples with respect to normal tissues, which is consistent with an enhanced gene expression. Conversely, BIRC5 showed a higher promoter methylation level in tumors (Figure S2c). 

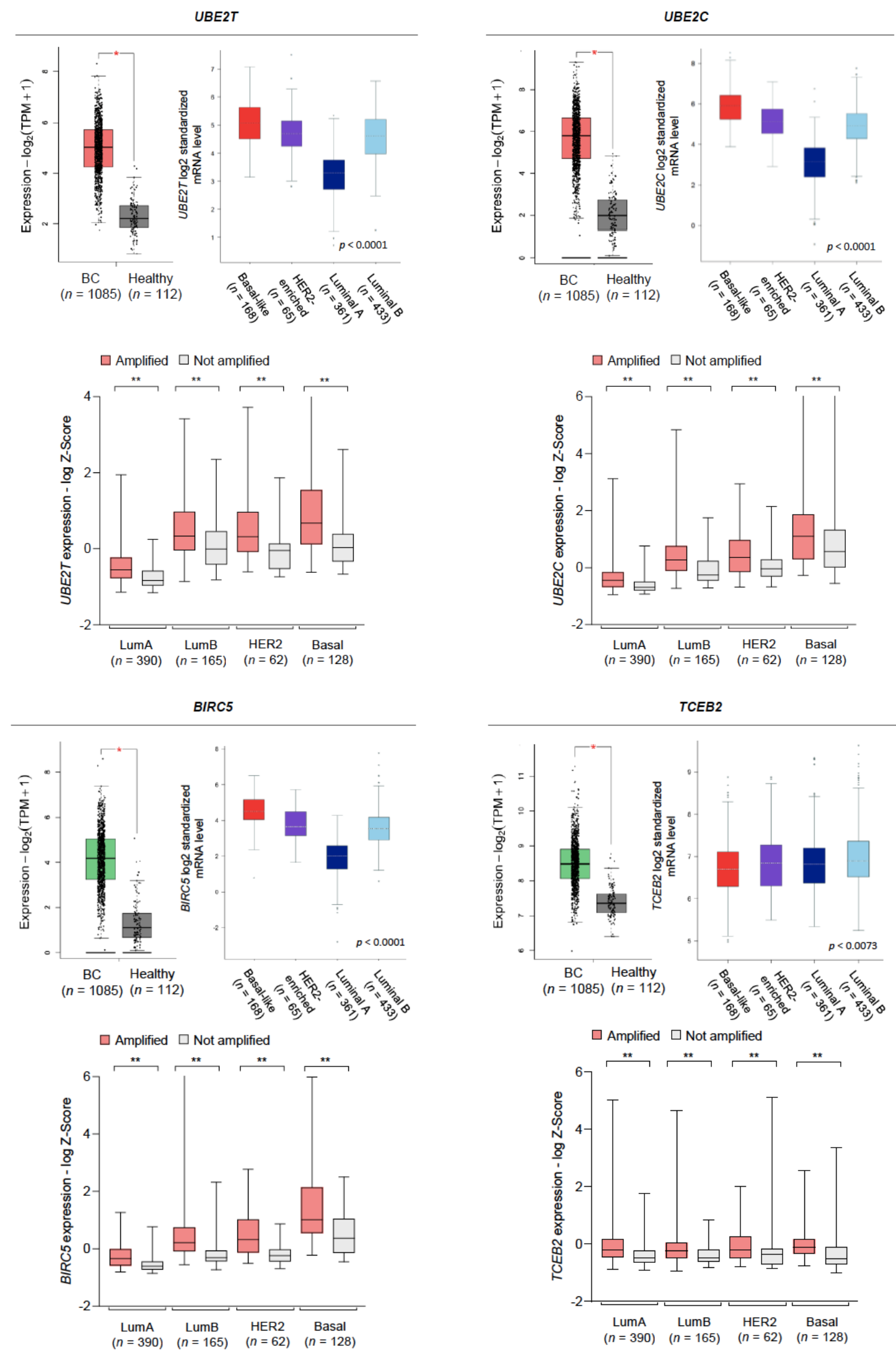

Figure 3. Expression levels of the most frequently amplified genes. Statistically significant differences in gene expression between tumor and healthy samples, along with the distribution of expression levels across intrinsic subtypes (upper part). Association of the amplification status with expression levels across intrinsic subtypes (lower part). Single asterisk denotes $p<0.05$ and double asterisk denotes $p<0.01$ using the Mann-Whitney test. 


\subsection{Amplification of SUN Genes Predicts an Unfavorable Outcome in Breast Tumors}

The KM plotter tool was used to explore the prognostic role of each gene amplification occurring in more than $2 \%$ of patients in both TCGA and METABRIC datasets [25]. High transcriptional levels of SUMO2, TCEB2, BIRC5, UBE2T, DERL1, PRKDC, and UBE2C, were associated with a shorter relapse-free survival (RFS) and their Kaplan-Meier curves are displayed in Figure 4a. Further, we validated these results by interrogating the METABRIC and TCGA datasets and found that BIRC5, UBE2T, and UBE2C remained significantly linked to a worse RFS with a false discovery rate (FDR) $<5 \%$ for almost all associations (Table S1a; clinicopathological features of patients from TCGA and METABRIC datasets with survival data are summarized in Table S1b). Stratification by nodal involvement and histological grade did not substantially modify the association with outcome, although most analysis presented an FDR $>10 \%$, probably due to the limited population sample in each group (Table S1c). Only BIRC5, UBE2T and UBE2C remained significantly linked to a worse RFS with an FDR of $1 \%$ in the subgroup of node positive patients. Interestingly, most of these amplifications were co-occurrent within the same tumors, displaying a strong statistical association (Figure $4 b$ ), and yet cytogenetic mapping showed that only BIRC5 and SUMO2, and DERL1 and PRKDC, are located in the same chromosomes and arms (17q25.3 and 17q25.1, and 8q24.13 and 8q11.21, respectively). The STRING database was used to map their interaction network revealing a significant enrichment $p$-value and low-to-moderate local clustering coefficient, defined as the likelihood of the connection (Figure 4c).

(a)
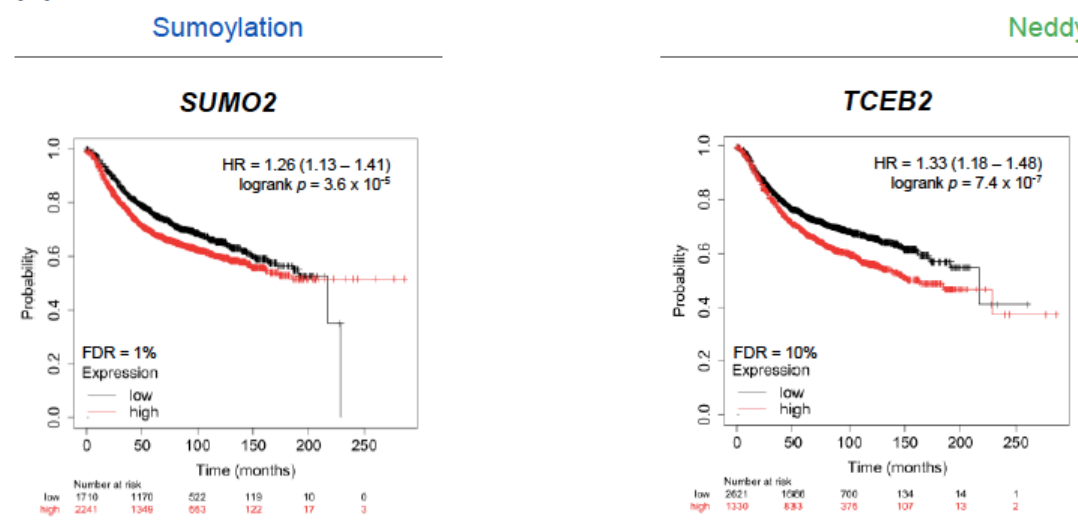

eddylation

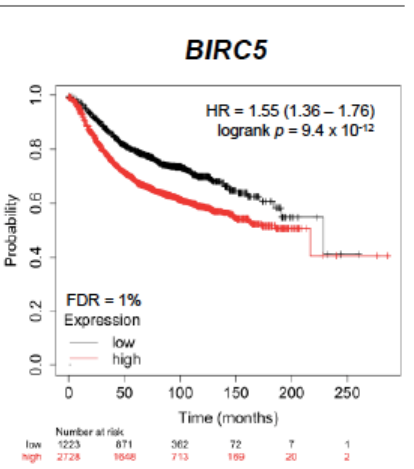

Ubiquitination
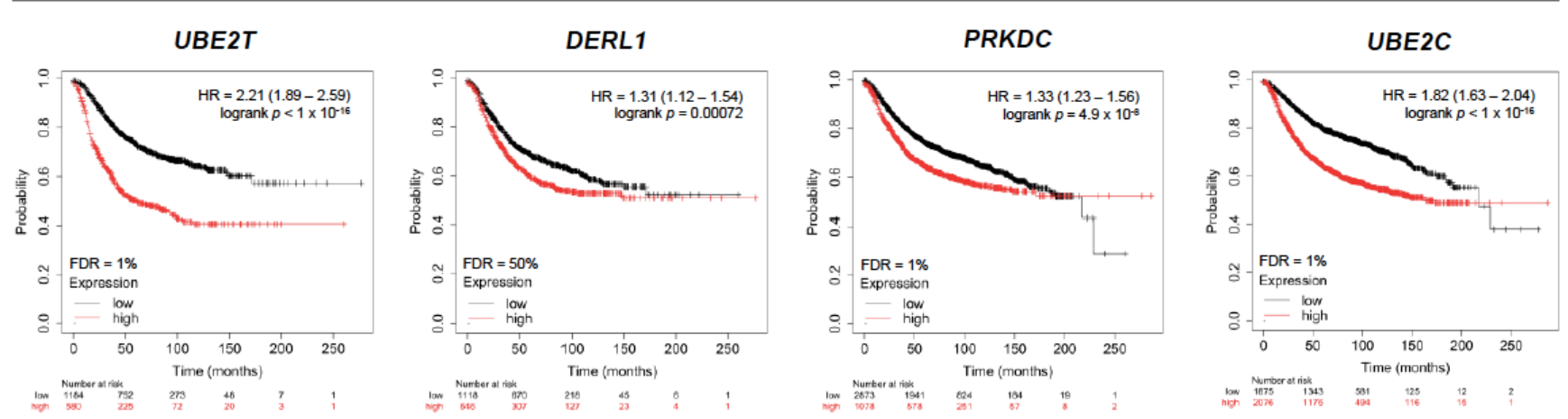

Figure 4. Cont. 
(b)

\begin{tabular}{ccccccc} 
A & B & $\begin{array}{c}\text { A not B/B } \\
\text { not A }\end{array}$ & Both & Log2OR & q-value & Tendency \\
\hline SUMO2 & BIRC5 & $41 / 44$ & 131 & $>3$ & $<0.001$ & Co-occurrence \\
& DERL1 & $99 / 582$ & 73 & 1.502 & $<0.001$ & Co-occurrence \\
& UBE2C & $156 / 103$ & 16 & 1.434 & 0.002 & Co-occurrence \\
PRKDC & UBE2C & $283 / 93$ & 26 & 1.354 & $<0.001$ & Co-occurrence \\
TCEB2 & PRKDC & $142 / 256$ & 53 & 1.888 & $<0.001$ & Co-occurrence \\
& UBE2T & $132 / 545$ & 63 & 0.978 & $<0.001$ & Co-occurrence \\
& DERL1 & $132 / 592$ & 63 & 0.828 & $<0.001$ & Co-occurrence \\
DERL1 & PRKDC & $384 / 38$ & 271 & $>3$ & $<0.001$ & Co-occurrence \\
& UBE2C & $598 / 62$ & 57 & 1.804 & $<0.001$ & Co-occurrence \\
\multirow{2}{*}{ BIRC5 } & DERL1 & $100 / 580$ & 75 & 1.530 & $<0.001$ & Co-occurrence \\
& UBE2C & $155 / 99$ & 20 & 1.823 & $<0.001$ & Co-occurrence \\
& PRKDC & $148 / 282$ & 27 & 0.712 & 0.034 & Co-occurrence \\
\hline
\end{tabular}

(c)

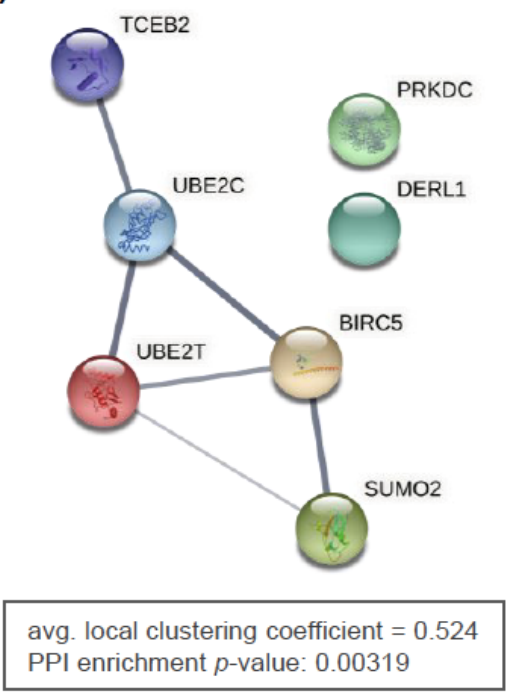

Figure 4. Association between gene expression and a worse relapse-free survival in breast cancer patients. (a) Survival analysis for individual genes correlated with poor relapse-free survival among the frequently amplified genes. (b) Cooccurrence of mutations in the analyzed population calculated by the odds ratio method. (c) Protein-protein interaction map displaying the significant functional network integrated by the selected genes. Line thickness denotes the strength of the association.

2.4. Amplification of UBE2T, UBE2C, and BIRC5 Genes Is Associated to a Worse Prognosis in Luminal and Basal-Like Breast Tumors

In order to further filter for potential clinical relevance, we next investigated the prognostic implication of those amplified genes across intrinsic subtypes. Despite showing a consistent prognostic capability, most of them did not pass a restrictive screening excluding those not reaching statistical significance, with an FDR over 5\%, and/or with low clinical impact (defined as a HR of RFS < 1.5) (Figure 5a). High levels of UBE2T predicted a poorer RFS in luminal A/B and basal-like tumors, whereas UBE2C and BIRC5 associated with a worse outcome in luminal A/B and luminal A tumors, respectively (Figure 5b). Validation in the METABRIC and TCGA datasets showed consistent results, with the exception of a loss of statistical significance of $U B E 2 T$ in basal-like tumors and overall higher FDRs as a result of the reduced population available (Table S2).

(a)

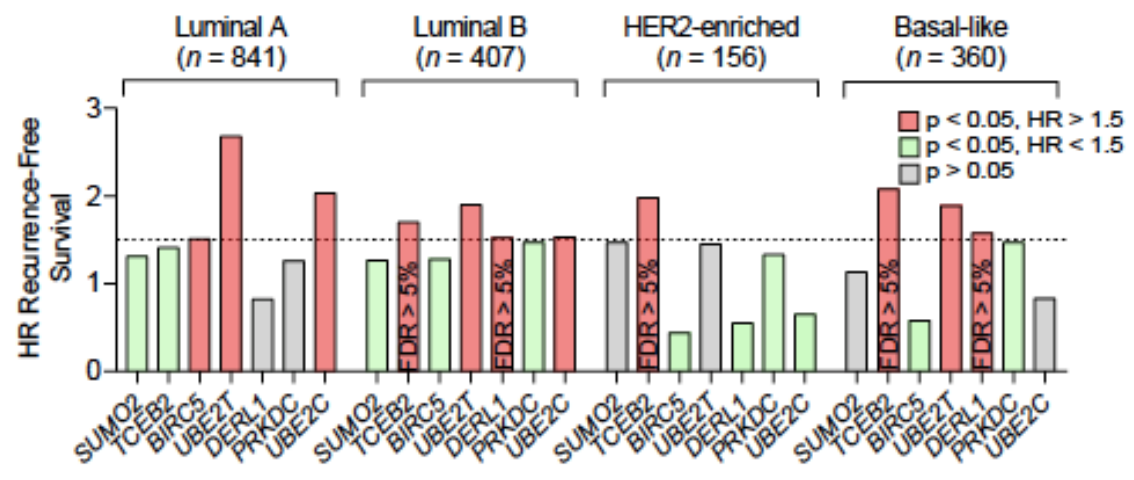

Figure 5. Cont. 
(b)

UBE2T
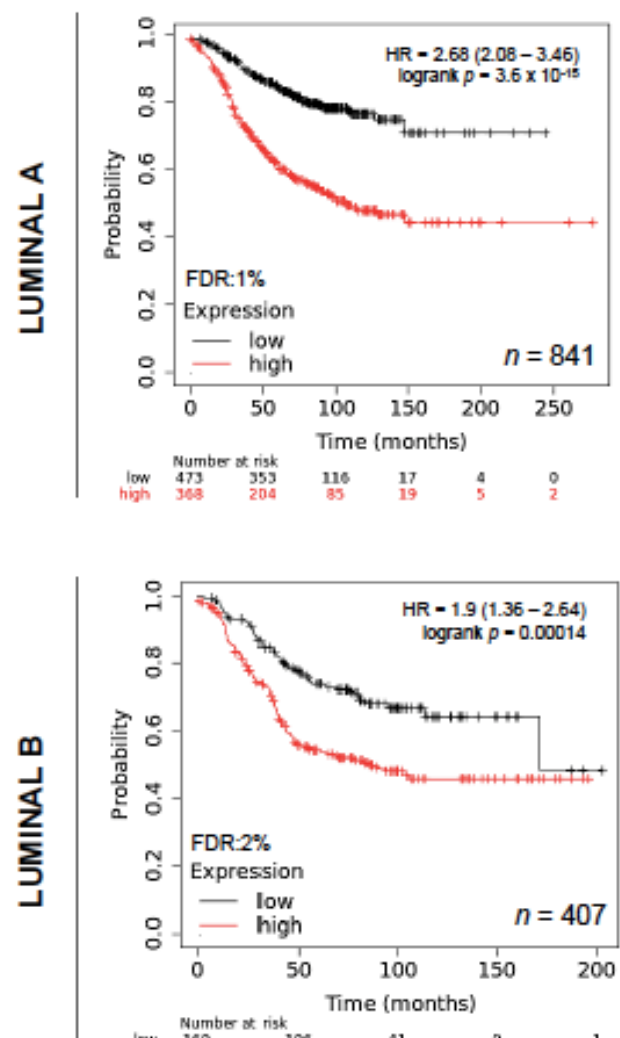

$\begin{array}{lll}\text { low } & \text { Number ot risk } \\ \text { high } & 100 \\ 247 & 1106\end{array}$

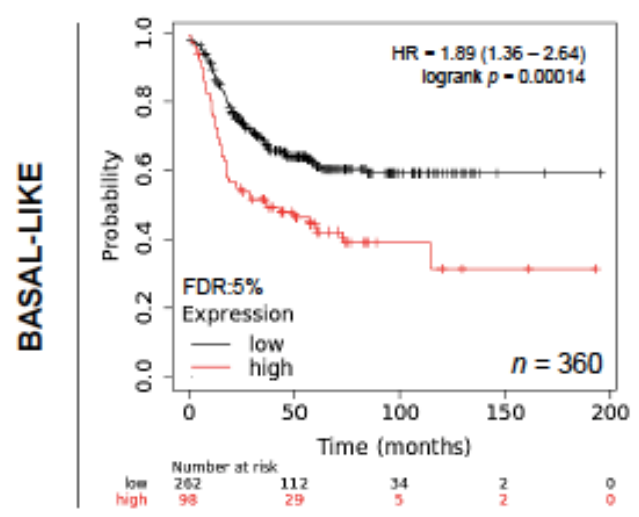

Neddylation

BIRC5
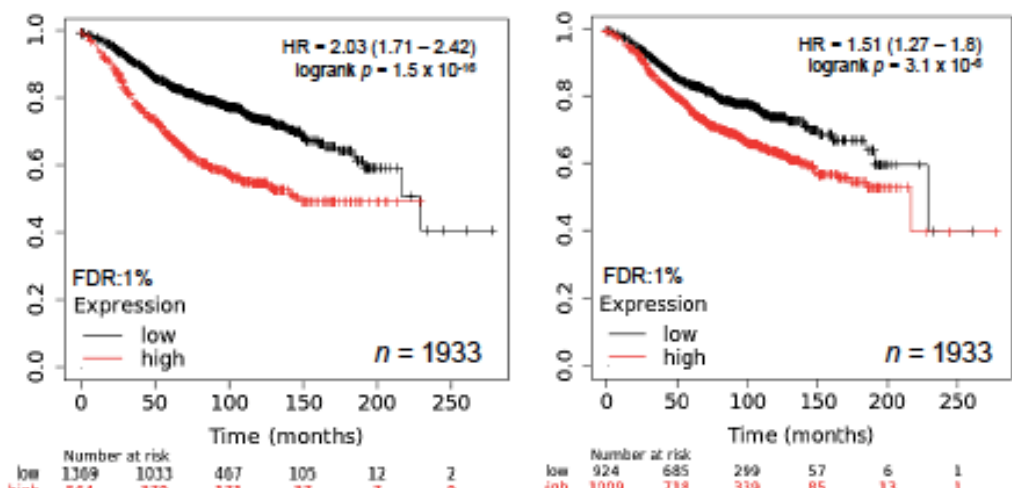

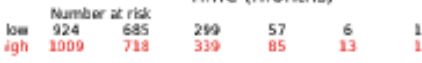

Figure 5. Association between gene expression and a worse relapse-free survival across intrinsic subtypes of breast cancer. (a) Filtering of gene amplifications with prognostic capability using the following criteria: statistical significance $p<0.05$, FDR $<5 \%$, HR of RFS $>1.5$. (b) Survival analysis for the selected genes correlated with poor relapse-free survival across different intrinsic subtypes of breast cancer.

2.5. Amplification of UBE2T, UBE2C, and BIRC5 Genes Can Predict Response to (neo)adjuvant Therapy in Luminal $A$ and Basal-Like Tumors

The ROCplot online tool was used to evaluate whether higher transcriptional levels of $U B E 2 T, U B E 2 C$, and $B I R C 5$, could qualify as putative biomarkers of response to 
(neo)adjuvant therapy in BC [26]. In the neoadjuvant setting, UBE2T performed substantially well as a surrogate of a worse rate of pathological complete response (pCR) after chemotherapy in TNBC (AUC 0.629, chi-square $p=0.028$ ) (Figure 6a). In the adjuvant setting, a higher expression of $U B E 2 C$ and $B I R C 5$ predicted a poorer response in luminal A patients treated with chemotherapy (AUC 0.796, chi-square $p=8.9 \times 10^{-5}$, and AUC 0.737 , chi-square $p=0.017$, respectively), although the limited number of evaluable patients calls for a cautious interpretation and further validation (Figure 6b). A higher expression of UBE2C and BIRC5 also significantly, although slightly, correlated with a shorter RFS in luminal A patients receiving adjuvant hormone therapy (AUC 0.588, chi-square $p=0.0093$, and AUC 0.577, chi-square $p=0.024$, respectively) (Figure 6c). A control analysis was performed assessing the expression of $E S R 1$, a clinically established biomarker predictive of response to endocrine therapy. A combined signature of $U B E 2 C$ and BIRC5 was statistically significant in both scenarios but did not increase the predictive capability.

(a)

TNBC

pCR after neoadjuvant chemotherapy
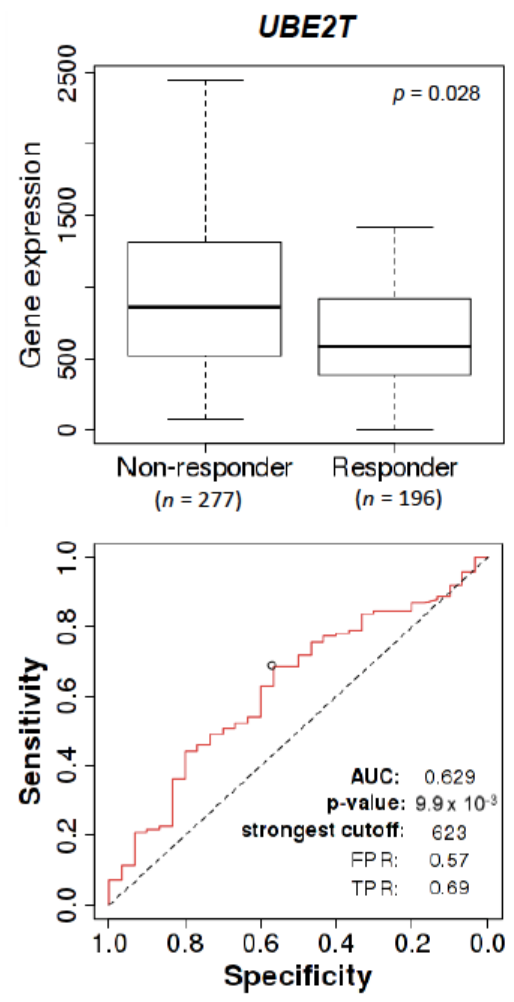

(b)

Luminal $A$

RFS at 5 years with adjuvant chemotherapy
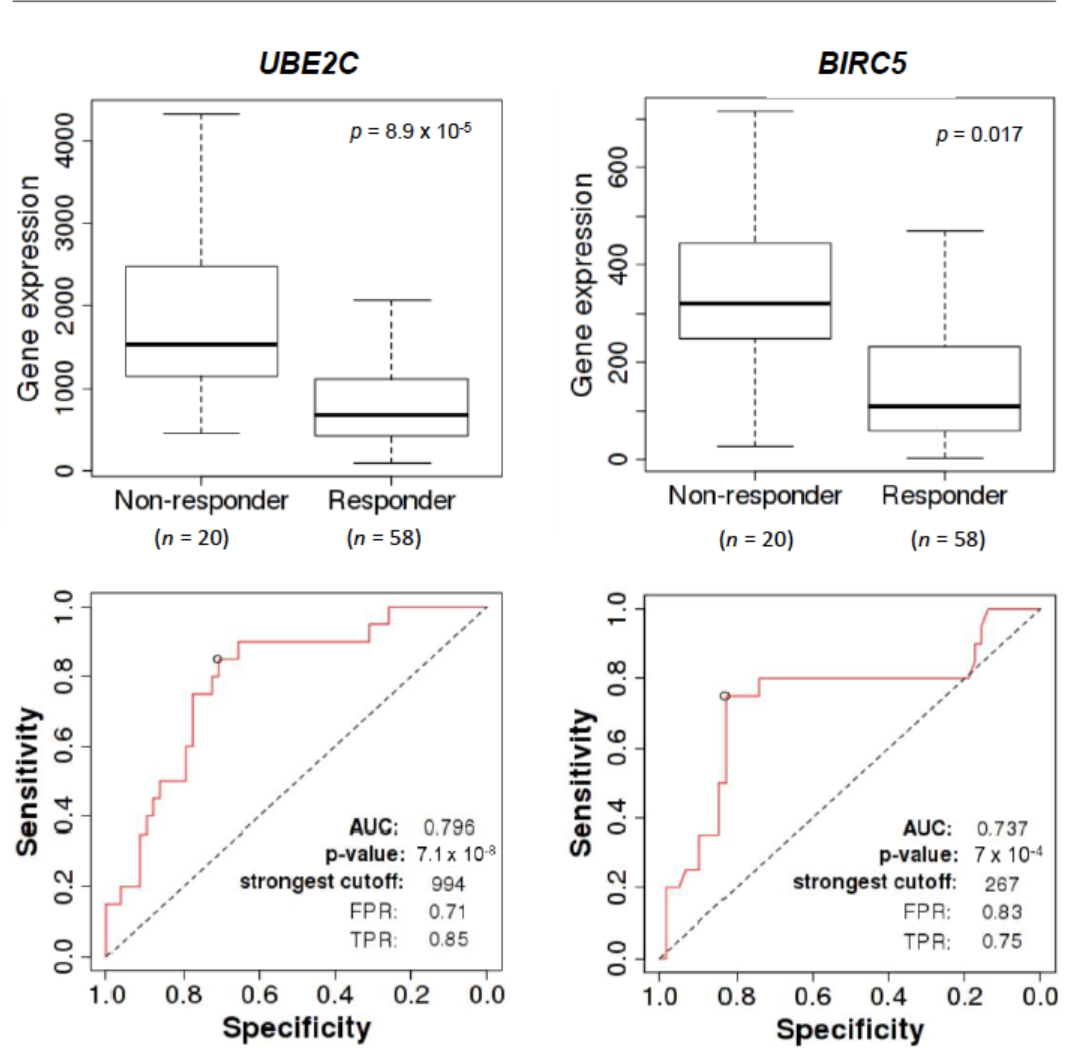

Figure 6. Cont. 
(c)

\section{Luminal A}

RFS at 5 years with adjuvant endocrine therapy
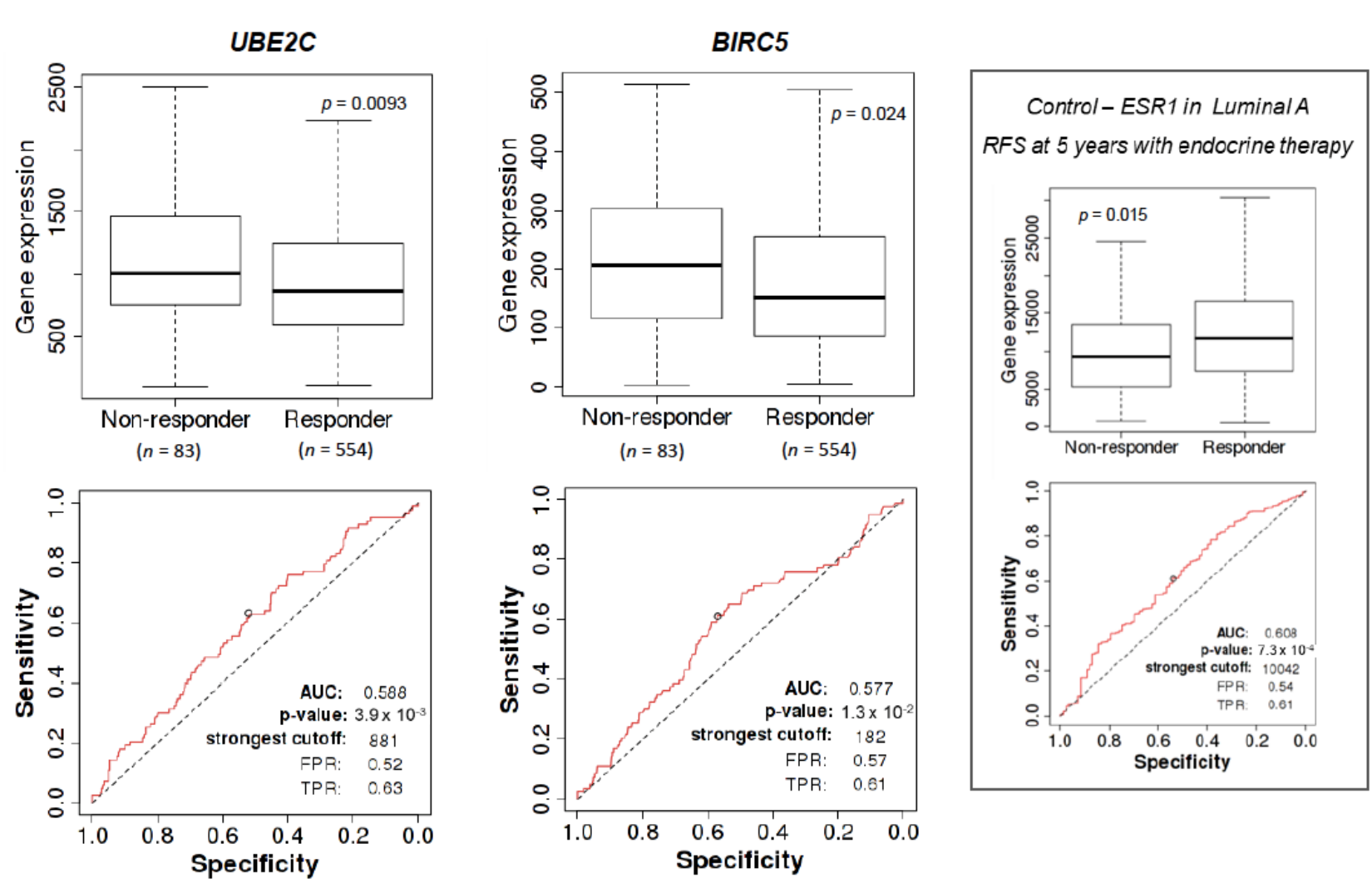

Figure 6. Association between gene expression and response to anti-tumor therapies. (a) Difference in gene expression of $U B E 2 T$ in TNBC patients according to the achievement of a pathological complete response (pCR) after neoadjuvant therapy. ROC curve displaying the predictive capability of pCR of UBE2T. (b) Difference in gene expression of UBE2C and BIRC5 in luminal A patients according to the achievement of 5 years of RFS after adjuvant chemotherapy. ROC curve displaying the predictive capability of 5 years RFS of UBE2C and BIRC5. (c) Difference in gene expression of UBE2C and BIRC5 in luminal A patients according to the achievement of 5 years of RFS with adjuvant endocrine therapy. ROC curve displaying the predictive capability of 5 years RFS of UBE2T and BIRC5. Companion graphs for ESR1 are included as control and help apprehend the potential clinical relevance of the predictive associations.

\subsection{Mapping of Mutations Occurring in SUN Genes and Prognostic Impact}

Although mutations in SUN components were not common, we explored their landscape in TCGA to further characterize their potential clinical interest (Figure 7a). Mutations occurring in more than $0.5 \%$ of patients were compared across intrinsic subtypes and their prognostic role was evaluated using the Genotype2Outcome tool (Figure $7 \mathrm{~b}, \mathrm{c}$ ) [27]. The transcriptional signature of each gene mutation was calculated as described in Material and Methods. The signature associated to USP9X predicted a worse overall survival in luminal A, HER2-enriched, and basal-like tumors, while that linked to USP7 predicted a worse outcome in luminal A tumors (Figure 7c). Figure 7d shows the top genes ranked by $p$-value for USP9X and USP7, respectively. The distribution of up- and downregulated genes contributing to the signature and the individual HR of RFS of the top ones ranked by $p$-value are displayed in Figure S3. Using the same dataset, we explored the type of mutation described in these genes (Table S3). A total of 13 patients had USP9X mutations, from which 9 were missense and 5 were truncating, whereas 6 patients had USP7 mutations, from which 5 were missense and 1 was truncating. No hotspots were observed. Only a 
single patient presented mutation co-occurrence, a trend which was not significant ( $\log 2$ $\mathrm{OR}>3, \mathrm{q}=0.092$ ). Online tools predicting the functional effects of these mutations (i.e., SIFT, PolyPhen, MutationTester, FATHMM-MKL) agreed in the deleterious impact of 2 missense mutations in USP7 (c.3011G >A and c.1223G $>$ A) and of 5 missense mutations in USP9X (c283G>C, c.1969C > T, c2225G>A, c.3894G >C, and c.6586C >G), none of which are archived in the ClinVar database. Although not described in the tools above mentioned, a total of 3 frameshift mutations occurring early in USP9X (c.273_274del, c.2084_2090del, and c.4104_4105del) are likely to be deleterious, as well as 1 stop mutation occurring in each USP9X (c.1861C $>$ T) and USP9X (c.2248C $>$ T). A single mutation of a donor splice site (c.5331+1G>A) was also identified and predicted to result in the loss of function of that site using the MaxEntScan tool, but its impact on USP9X activity remains unknown.

\subsection{Exploring Genomic Vulnerabilities in a Large Panel of Breast Cancer Cell Lines}

Finally, we searched for putative in vitro models that could help characterize the molecular underpinnings of the genomic alterations identified from patient data. To this end, we interrogated the Cancer Dependency Map (DepMap) portal, the Catalogue of Somatic Mutations in Cancer (COSMIC), and at the Cancer Cell Line Encyclopedia (CCLE), as described in Materials and Methods [28-31]. In contrast to the high prevalence of $U B E 2 T$, UBE2C, and BIRC5 amplifications in BC patients, only 2/49 cell lines (JIMT- 1 and HCC1569) harbored UBE2C amplifications and 3/49 cell lines (HCC1143, OCUB-M, and MDA-MB361) harbored BIRC5 amplifications (Figure 8a; Table S4). The copy number of these genes positively correlated with mRNA levels, and transcript levels were strongly associated with Ki67 expression, which is consistent with a more proliferative phenotype and a poorer prognosis (Figure $8 \mathrm{~b}, \mathrm{c}$ ). Expression of UBE2T, UBE2C and BIRC5 distributed across intrinsic subtypes in a similar fashion to $B C$ patients, with a tendency to be highest in basal-like cells and lowest in luminal cells. However, this trend lacked statistical significance, likely due to the limited sample size (Figure 8d). More cell lines presented mutations in USP9X $(8 / 49)$ and USP7 (5/49), although an unexpected high frequency of USP9X mutations in luminal cell lines was found along with a poor overall concordance with the mutational profile of patients. The predictive capability of the set of gene amplifications was explored using data from comprehensive online repositories (see Materials and Methods). No robust associations were observed, with varying trends among databases and reduced sample sizes as a result of our effort to stratify cell lines according to their intrinsic subtypes to recapitulate the actual clinical setting (Figures S4 and S5). 
(a)

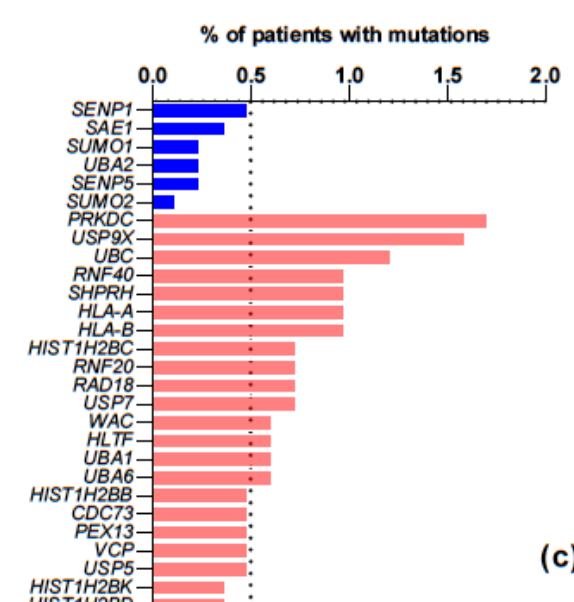

(c)

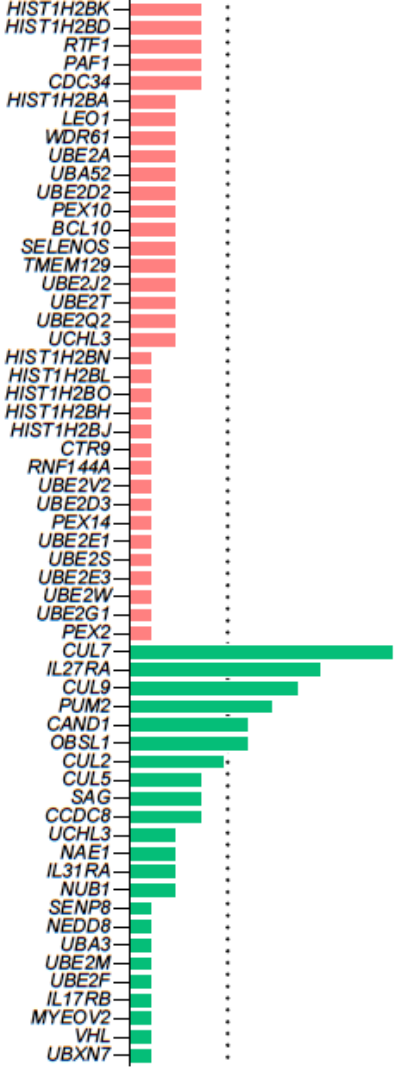

(d) (b)
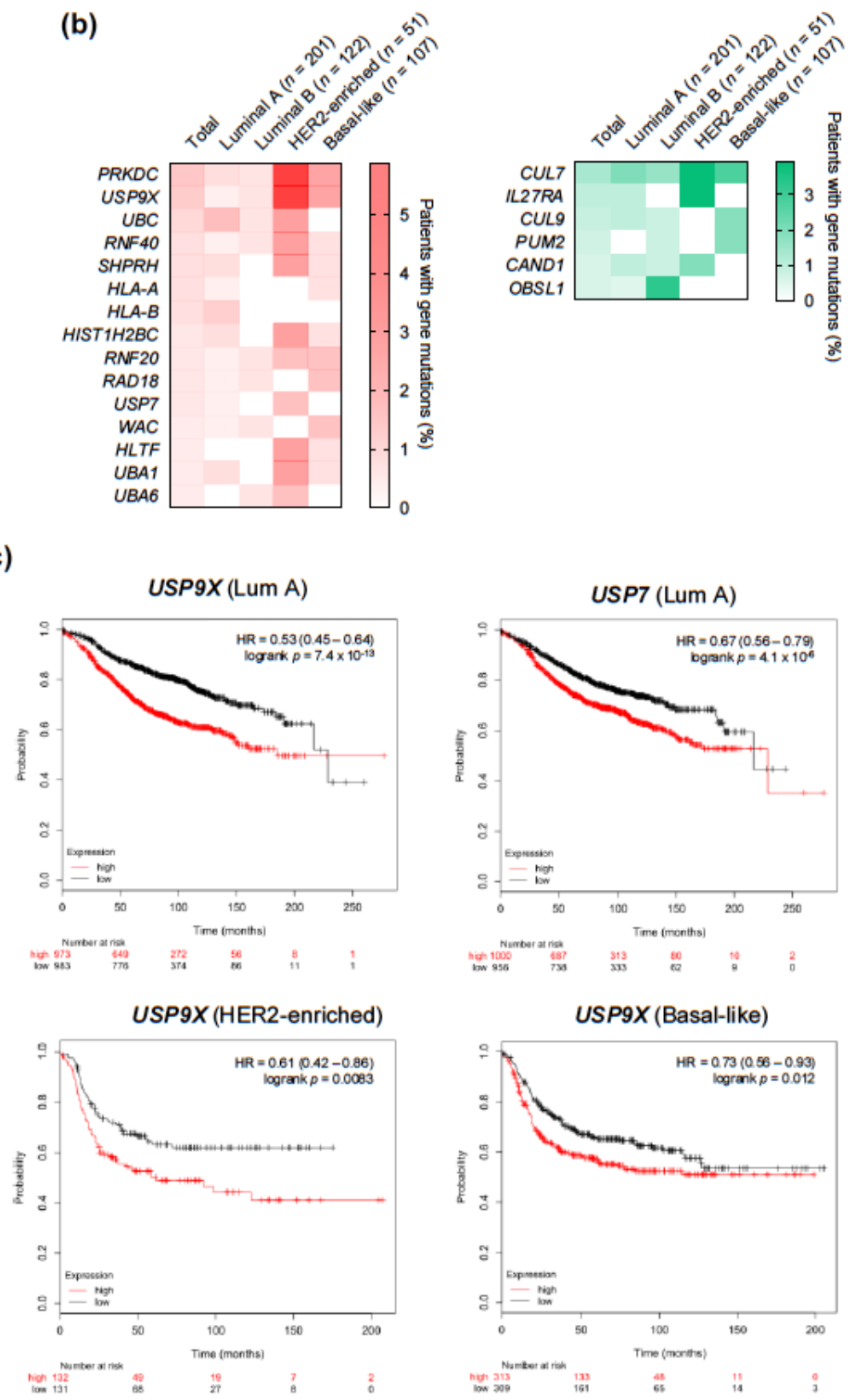

USP9X (Basal-like)
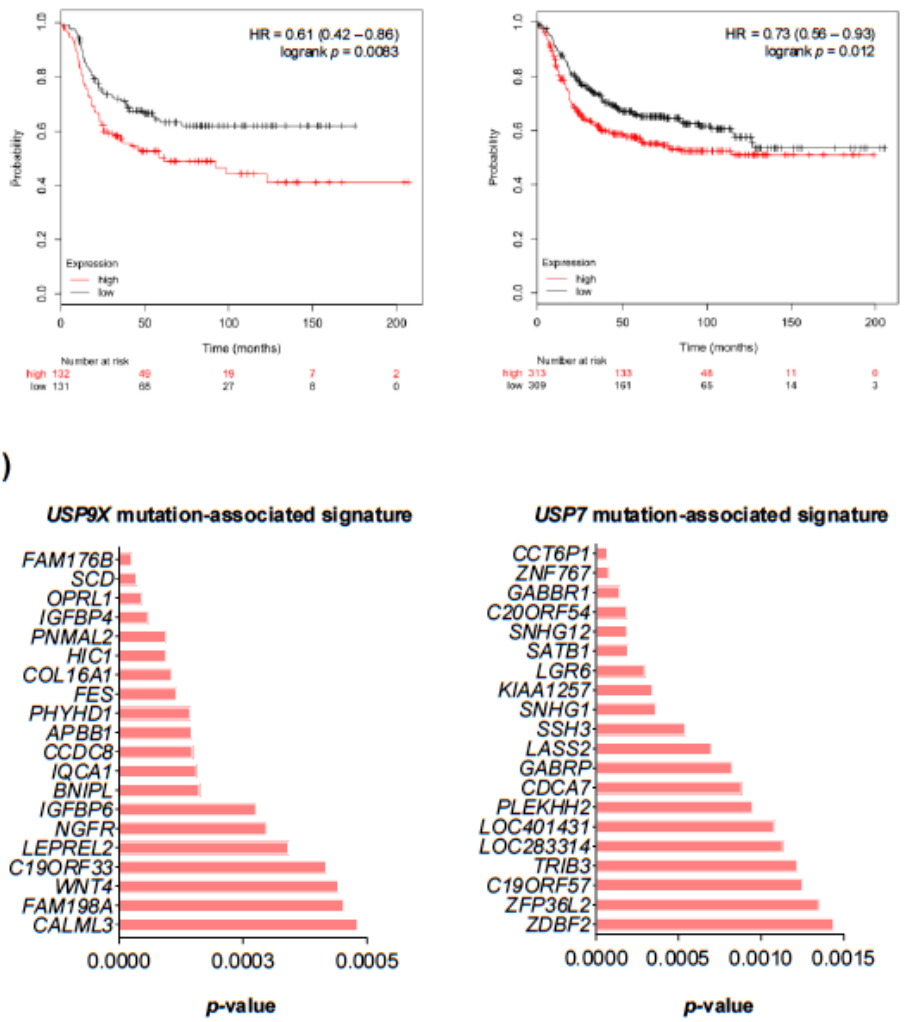

USP7 mutation-associated signature

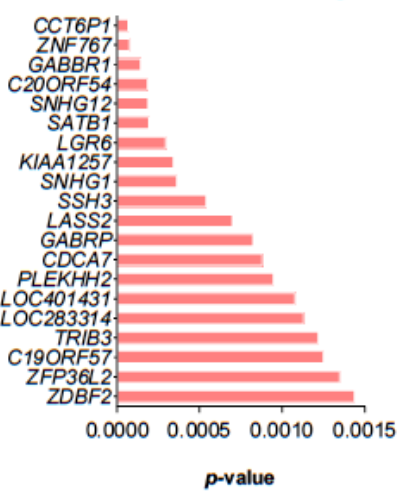

Figure 7. Mutational landscape and prognostic implications. (a) Frequency of gene mutations in breast cancer patients from the TCGA dataset. (b) Distribution of the frequencies of gene mutations over $0.5 \%$ across intrinsic subtypes. Population is limited to those patients in which the intrinsic subtype is available in cBioPortal for stratification. (c) Significant associations between the transcriptomic signatures of USP9X and UP7 mutations and overall survival across intrinsic subtypes. (d) Top USP9X and USP7-associated transcriptomic signature components, sorted by $p$-value. 
(a)

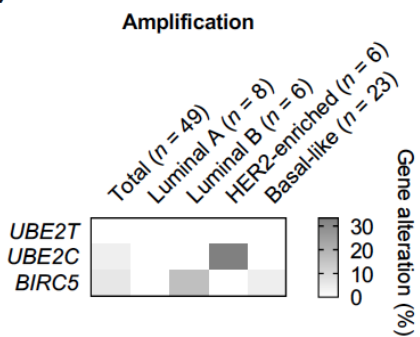

(b)

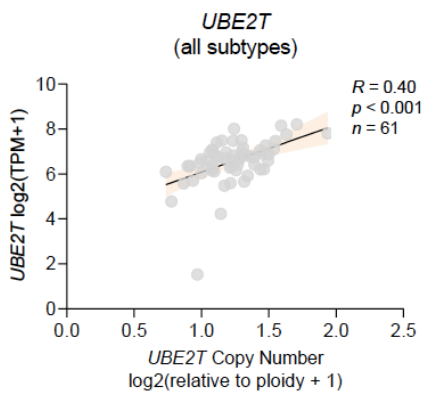

(c)
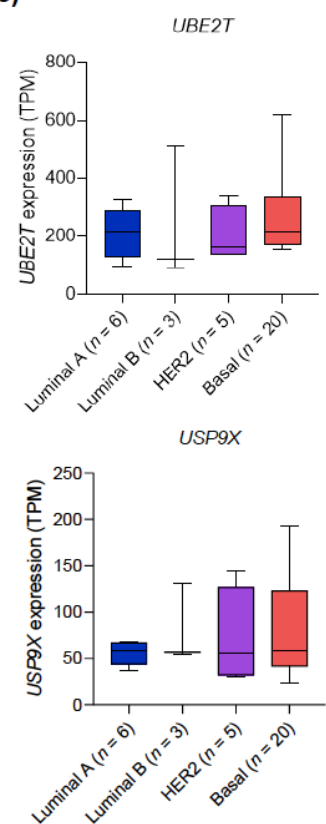

(d)

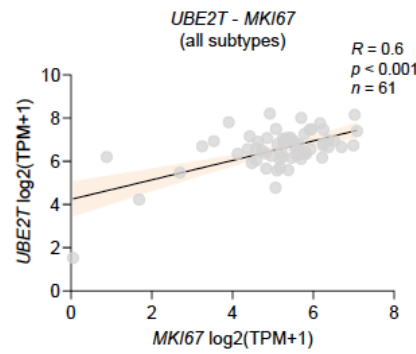

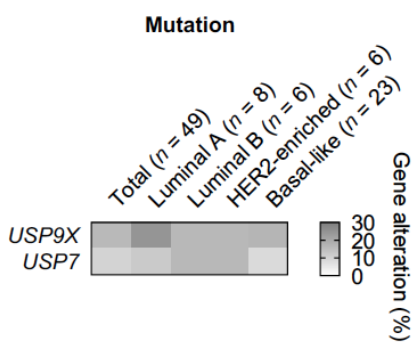

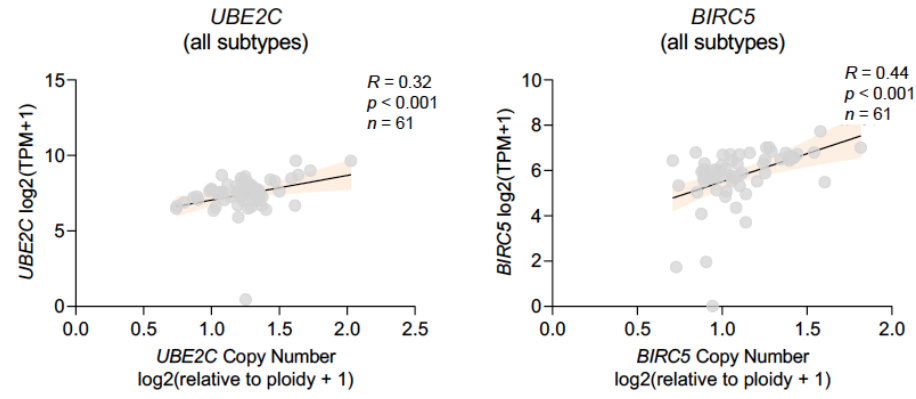

UBE2C

$B I R C$

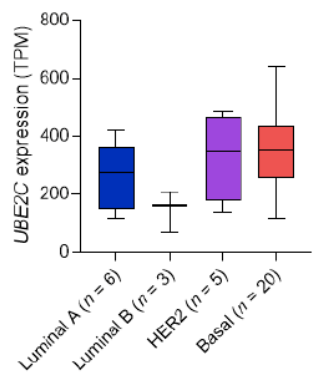

USP7

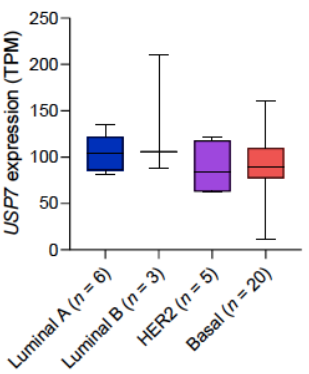

UBE2C - MKI6
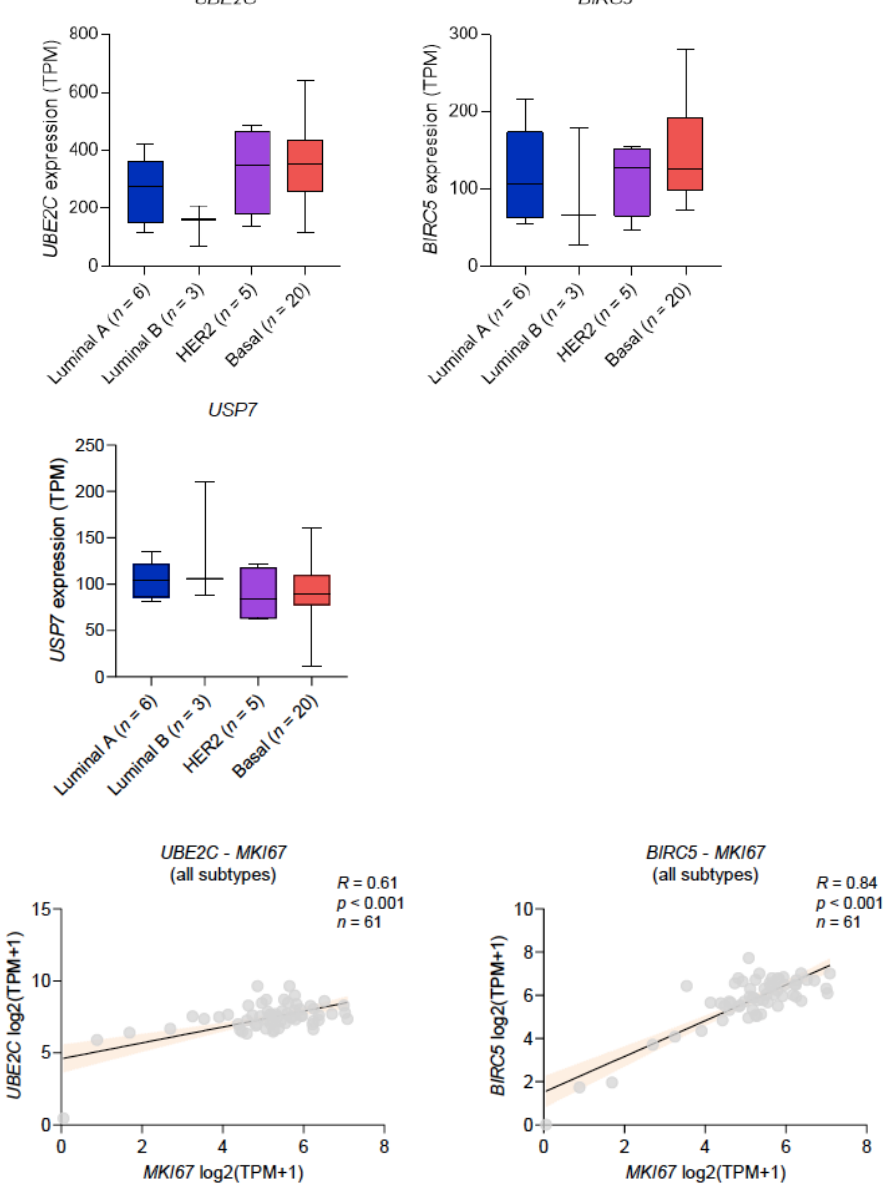

Figure 8. In vitro correlates of the genomic vulnerabilities identified in the SUN machinery in BC. (a) Heatmap showing the frequency of UBE2T, UBE2C, and BIRC5 amplifications, and USP9X and USP7 mutations, across cell lines of different intrinsic subtypes. (b) Positive dependance between copy number and gene expression in BC cell lines. (c) Distribution of gene expression across cell lines of the different intrinsic subtypes. (d) Positive correlation between the expression levels of UBE2T, UBE2C, and BIRC5, and proliferation marker MKi67. 


\section{Discussion}

In the present article we describe common genomic alterations in SUN genes in BC. By means of a comprehensive bioinformatic pipeline, the main goal of this work was to identify those genes linked to a worse prognosis and that therefore could be explored in the future as druggable targets and/or predictors of response to treatment.

By using TCGA and METABRIC datasets (combined $n=2989$ ), we observed that amplifications are by far the most frequent genomic alteration occurring in SUN genes. We further identified five, 15 , and six genes that presented a frequency of gene amplifications of more than $2 \%$ in SUMOylation, ubiquitination, and neddylation pathways, respectively. We refined our search by selecting only those with more than $2 \%$ of gene amplifications in both databases individually, thus reducing the list to four, 11 and four for the abovementioned processes, respectively. The filtered genes were then stratified across the different intrinsic subtypes. The mRNA levels of amplified genes tended to be higher in breast tumors than in the healthy state, although this difference reached statistical significance only for $U B E 2 T, B I R C 5, U B E 2 C$, and TCEB2. Also, with the exception of TCEB2, gene expression and protein levels tended to be highest in basal-like tumors and lowest in luminal A tumors. Overall, gene amplification led to higher transcriptional levels across subtypes. We may acknowledge that although the frequency of genomic alterations is useful to uncover potential molecular vulnerabilities, the magnitude of the amplification of each gene is better represented by the amount of mRNA levels, and thus the prognostic and predictive correlations shown in our work are based on expression levels. Identification of tumors harboring amplifications is easier to be implemented in the clinical setting as has been the case in BC for HER2. Of note, many genes that are not frequently amplified but may have high expression rates and be of prognostic interest in $B C$ are not discussed in our analysis. From the set of genes, SUMO2, TCEB2, BIRC5, UBE2T, DERL1, PRKDC, and UBE2C showed a significant association with a worse RFS. Co-occurrence of gene amplifications was commonly found along with a robust functional network. Relevant associations (HR of RFS >1.5) remained statistically significant and with an FDR $\leq 5 \%$ for UBE2T, UBE2C, and BIRC5, in luminal A/B and basal-like tumors, luminal A/B tumors, and luminal A tumors, respectively. Most of these results were recapitulated in the METABRIC and TCGA databases as validation. When exploring the predictive capability of these amplified genes, UBE2T expression levels were found significantly increased in TNBC patients not achieving pCR after neoadjuvant chemotherapy, whereas UBE2C and BIRC5 expression were higher in luminal A patients with tumor relapse within 5 years of endocrine therapy or chemotherapy.

Finally, we sought to characterize the mutational landscape and prognostic implications of the SUN components, and found that mutated USP9X and USP7 genes, analyzed through their surrogate transcriptomic signatures, were linked to a worse prognosis in luminal A, HER2-enriched, and basal-like tumors, and in luminal A tumors, respectively. Main findings and potential therapeutic approaches are outlined in Table 1.

Establishing a bioinformatic pipeline for omic analysis has progressively become a critical need for the discovery of therapeutic targets in cancer. These tools, guided by clinical experience, can provide intuitive access to a growing pool of data and generate fast and robust answers to experiments without inefficiently compromising patients. We showed that ubiquitin-conjugating enzymes E2T and E2C, and baculoviral IAP repeat containing 5 (BIRC5; also known as survivin) are amplified in BC and associated to a worse prognosis across subtypes and stages. Importantly, genomic amplifications correlated with increased mRNA levels. UBE2T and UBE2C have been previously associated with cancer progression and poor outcome in several solid tumors, although genomic evidence in $B C$ is recent and limited [32-36]. Consistently, in vitro functional experiments in ER positive HER2 negative $\mathrm{BC}$ cells showed that $U B E 2 C$ expression is a tumorigenic factor, that it is regulated by estrogen through direct binding to the UBE2C promoter region, and that its overexpression leads to estrogen-independent growth [37]. Moreover, UBE2C depletion markedly increased the cytotoxicity of letrozole, tamoxifen, doxorubicin, and 
the sensitivity to radiation therapy in multiple $B C$ cell lines $[37,38]$. In this regard, we showed that higher UBE2C mRNA levels are not only a prognostic factor but also predictive of a worse response to adjuvant endocrine therapy and chemotherapy in patients with luminal A tumors. Less explored so far, our group recently reported first evidence that $U B E 2 T$ is amplified in BC and non-small cell lung carcinomas, in which it was related to a detrimental outcome [36]. In the present work we describe that UBE2T predicts a worse outcome in luminal A/B and basal like tumors, and also a poorer response to neoadjuvant chemotherapy in TNBC. As a hint for its molecular rationale, UBE2T overexpression has been found to facilitate cell cycle progression and to avoid DNA repair by degrading key regulators of both functions such as p21 or BRCA1 in vitro and in vivo in BC [39]. This could also pave the way to exploring the benefit of PARP inhibitors in patients harboring somatic UBE2T overexpression independent of their BRCA status.

Table 1. Summary of genomic vulnerabilities of the SUN machinery with prognostic and predictive implications and potential therapeutic avenues.

\begin{tabular}{|c|c|c|c|c|c|c|c|c|c|}
\hline $\begin{array}{c}\text { Gene } \\
\text { (Process) }\end{array}$ & Alteration & $\begin{array}{l}\text { Prognostic } \\
\text { Implication }\end{array}$ & $\begin{array}{l}\text { Intrinsic } \\
\text { Subtype }\end{array}$ & $\begin{array}{l}\text { Predictive } \\
\text { Implication }\end{array}$ & $\begin{array}{l}\text { Intrinsic } \\
\text { Subtype }\end{array}$ & $\begin{array}{l}\text { Previous } \\
\text { Clinical } \\
\text { Evidence }\end{array}$ & $\begin{array}{l}\text { Previous } \\
\text { Preclinical } \\
\text { Evidence }\end{array}$ & $\begin{array}{c}\text { Potential } \\
\text { Therapeutic } \\
\text { Avenues }\end{array}$ & Refs. \\
\hline $\begin{array}{c}\text { UBE2T } \\
\text { (Ubi) }\end{array}$ & Amp & $\begin{array}{l}\text { Poorer } \\
\text { survival }\end{array}$ & $\begin{array}{l}\text { Luminal } \\
\text { Basal-like }\end{array}$ & $\begin{array}{c}\text { Resistance to } \\
\text { neoadjuvant } \\
\text { Cx }\end{array}$ & Basal-like & $\begin{array}{c}\text { shorter RFS } \\
\text { (basal-like) }\end{array}$ & $\begin{array}{c}\text { Impairment of } \\
\text { DNA repair } \\
\text { (FANCD2, } \\
\text { BRCA1 } \\
\text { degradation) }\end{array}$ & $\begin{array}{c}\text { - Targeted } \\
\text { inhibition. } \\
\text { - Combination } \\
\text { strategies (Cx } \\
\text { PARPi) }\end{array}$ & {$[36,39]$} \\
\hline $\begin{array}{c}\text { UBE2C } \\
\text { (Ubi) }\end{array}$ & Amp & $\begin{array}{l}\text { Poorer } \\
\text { survival }\end{array}$ & Luminal & $\begin{array}{c}\text { Resistance to } \\
\text { adjuvant ET } \\
\text { and Cx }\end{array}$ & Luminal & $\begin{array}{c}\text { Shorter RFS } \\
\text { and OS (all } \\
\text { subtypes) }\end{array}$ & $\begin{array}{l}\text { Resistance to } \\
\text { ET, Cx, and RT } \\
\text { (mitotic cyclins } \\
\text { degradation) }\end{array}$ & $\begin{array}{c}\text { - Targeted } \\
\text { inhibition. } \\
\text { - Combination } \\
\text { strategies (ET, } \\
\text { Cx, RT) }\end{array}$ & $\begin{array}{l}{[33-35,} \\
37,38]\end{array}$ \\
\hline $\begin{array}{l}\text { BIRC5 } \\
\text { (Nedd) }\end{array}$ & Amp & $\begin{array}{l}\text { Poorer } \\
\text { survival }\end{array}$ & Luminal & $\begin{array}{c}\text { Resistance to } \\
\text { adjuvant ET } \\
\text { and Cx }\end{array}$ & Luminal & $\begin{array}{c}\text { shorter } \\
\text { RFS/OS (all } \\
\text { subtypes) } \\
\text { shorter RFS } \\
\text { stage II/III } \\
\text { (all } \\
\text { subtypes) }\end{array}$ & $\begin{array}{l}\text { Apoptosis } \\
\text { inhibitor }\end{array}$ & $\begin{array}{c}\text { - Targeted } \\
\text { inhibition. } \\
\text { - Combination } \\
\text { strategies (ET, } \\
\text { Cx, PARPi) }\end{array}$ & [40-45] \\
\hline $\begin{array}{c}\text { USP9X } \\
\text { (Ubi) }\end{array}$ & $\begin{array}{c}\text { Mut } \\
\text { (deleterious) }\end{array}$ & $\begin{array}{l}\text { Poorer } \\
\text { survival }\end{array}$ & $\begin{array}{l}\text { Luminal } \\
\text { HER2 } \\
\text { Basal-like }\end{array}$ & - & - & - & $\begin{array}{c}\text { - BRCA1 } \\
\text { stabilization } \\
\text { - Sensitivity to } \\
\text { tamoxifen }\end{array}$ & $\begin{array}{l}\text { - Predictor of } \\
\text { resistance to } \\
\text { ET and } \\
\text { sensitivity to } \\
\text { Cx and PARPi } \\
\text { - Explore } \\
\text { actionability in } \\
\text { upregulated } \\
\text { signature }\end{array}$ & [46-49] \\
\hline $\begin{array}{l}\text { USP7 } \\
\text { (Ubi) }\end{array}$ & $\begin{array}{c}\text { Mut } \\
\text { (deleterious) }\end{array}$ & $\begin{array}{l}\text { Poorer } \\
\text { survival }\end{array}$ & Luminal & - & - & - & $\begin{array}{l}\text { Impairment of } \\
\text { mitotic } \\
\text { progression }\end{array}$ & $\begin{array}{c}\text { - Predictor of } \\
\text { sensitivity to } \\
\text { Cx } \\
\text { - Explore } \\
\text { actionability in } \\
\text { upregulated } \\
\text { signature }\end{array}$ & [50-52] \\
\hline
\end{tabular}

Ubi: ubiquitination; Nedd; neddylation; Amp: amplification; Mut: mutation; Cx: chemotherapy; ET: endocrine therapy; RFS: relapse-free survival; OS: overall survival; RT: radiotherapy; ER: estrogen receptor.

In addition to ubiquitin, the ubiquitin superfamily also contains ubiquitin-like proteins, including Nedd8 and SUMO, which do not only have sequence homology and structural similarity to ubiquitin but also use a similar enzymatic cascade to modify their substrate proteins [53]. We reported that BIRC5 amplification is a biomarker of a worse prognosis in luminal A patients, and also predicts a worse response to adjuvant endocrine therapy or chemotherapy in these patients. These findings are consistent with a recent in silico analysis by Dai et al. and with the observation by Hamy et al. that BIRC 5 predicts a worse RFS in stage II/III BC patients of all intrinsic subtypes who do not achieve a pCR after neoadjuvant chemotherapy [40,41]. Surprisingly, the promoter methylation level of BIRC5 was found to be increased in tumor samples, which appears to go against its expression pattern. Although this finding has not been clearly explained in BC, previous observations 
in endometrial cancer have found that the hypermethylation of the BIRC5 promoter blocks the binding of $\mathrm{p} 53$, a repressor of BIRC5 gene transcription, to its promoter region, thus increasing its expression [42,43]. Also interestingly, BIRC5 is one of the genes included in the Oncotype DX ${ }^{\circledR}$, Endopredict ${ }^{\circledR}$ and Prosigna ${ }^{\circledR}$ signatures. There is in vitro evidence that repressing BIRC5 expression by siRNA could significantly inhibit proliferation and also induce a BRCAness phenotype with DNA double-strand breaks and a functional impairment of homologous recombination [44,45]. A comprehensive review by Li et al. has recently gathered together the advances in cancer therapeutics targeting BIRC5 [54].

We also found that mutations in USP9X and USP7 have an ominous prognostic impact in luminal, HER2-enriched, and basal-like breast tumors. USP9X and USP7 are ubiquitin-specific proteases with roles somewhat antagonistic to UBE2T and UBE2C [55]. Consistently, inactivating missense and truncating mutations should recapitulate the effects of those amplifications. However, the molecular interplay underlying such clinical effects remains unclear. USP9X has been shown to stabilize BRCA1 and confer resistance to DNA-damaging agents, and yet its downregulation rendered BC cells resistant to tamoxifen, while its up-modulation has been found to promote centrosome amplification, chromosome instability, and higher histologic grades of $\mathrm{BC}$, as well as to enhance Hippo pathway-dependent cell proliferation [46-49]. The role of USP7, in turn, appears to be more established in the regulation of DNA replication and mitosis progression by affecting the stability of Aurora-A kinase and Geminin [50,51]. Of note, USP7 physically interacts with the $\mathrm{ER} \alpha$, thereby mediating its deubiquitination and stabilization [52]. Altogether, this body of evidence underscores the potential utility of studying USP9X, USP7, and their associated transcriptomic signatures especially in luminal BC, although further functional characterization of specific mutations is required.

Given the potential interest of therapeutically exploiting this set of genomic vulnerabilities and the need to further describe their molecular foundations, we conducted a comprehensive in silico characterization of BC cell lines using mutational, transcriptional, and sensitivity data. Very few cell lines, from a panel including the most typically used and thoroughly described, presented the genomic alterations identified in patients. Their distribution across intrinsic subtypes also differed, and drug sensitivity analyses showed only slight tendencies that did not reach statistical significance. Molecular and pharmacological studies will be hindered in the absence of robust in vitro models recapitulating these gene alterations. However, the low frequency of the genomic alterations in cell lines has also been observed in other vulnerabilities (e.g., MET mutations in lung cancer or BRCA1/2 in $B C)$, and this fact has not impacted the clinical development of strategies against them.

In sum, our data suggests that the amplification of UBE2T, UBE2C, and BIRC5, and inactivating mutations in USP9X and USP7, can predict a worse outcome in different intrinsic subtypes of BC. Comprehensive initiatives are currently focused on leveraging the prognostic implications of the ubiquitin and ubiquitin-like pathways in cancer. However, to the best of our knowledge, no targeted agents against UBE2T, UBE2C, BIRC5, or USP9X, either approved or in development, can be found in The Drug Gene Interaction Database or Genomics of Drug Sensitivity in Cancer database, while the only USP7 inhibitor, P22077, has not been tested in BC [56]. These findings are exploratory and require further validation in preclinical models and patient cohorts, particularly considering the influence of CDK4/ 6 inhibitors and newly approved targeted agents in BC, as well as further efforts to characterize the molecular and functional properties of the described alterations for drug design.

\section{Materials and Methods}

\subsection{Identification of SUN Genes Gene Ontology}

Reactome (https:/ / reactome.org/, accessed on: 8 January 2021), a publicly available relational database of signaling and metabolic molecules and their relations organized into biological pathways and processes, was used to sequentially narrow down genes involved the metabolism of proteins, post-translational modifications of proteins, and, 
finally, the processes of interest, that is, SUMOylation, ubiquitination, and neddylation. Further confirmation of the biological functions related to each gene set was obtained using the 2018 Molecular_function Gene Ontology Terms through the publicly available EnrichR online platform (https: / / maayanlab.cloud/Enrichr/, accessed on: 8 January 2021).

\subsection{Data Collection and Processing}

We used data contained at cBioportal (www.cbioportal.org, accessed on: 8 January 2021) from patients with breast invasive carcinoma to explore the distribution of copy number alterations (CNA) and mutations occurring in the selected genes. The TCGA (Cell 2015 database, $n=817)$ and METABRIC $(n=2509)$ datasets were interrogated, retrieving a total pool of 3326 patients with mutational data, 2989 patients with CNA data, and 2989 with both.

Transcription levels of the selected genes in breast tumors versus health controls were analyzed using the GEPIA2 web server (Gene Expression Profiling Interactive Analysis; http:/ / gepia2.cancer-pku.cn/, accessed on: 8 January 2021). GEPIA2 is an updated version of GEPIA for analyzing the RNA sequencing expression data of 9736 tumors and 8587 normal samples from the TCGA and the GTEx projects. The differences in mRNA levels across intrinsic subtypes were explored in the bc-GenExMiner v4.5. web tool, which comprises RNA-seq data from 4912 tumor samples from the TCGA, SCAN-B, and GTEx projects ( http:/ /bcgenex.centregauducheau.fr/, accessed on: 8 January 2021). The UALCAN web resource (http:/ / ualcan.path.uab.edu/, accessed on: 8 January 2021), which integrates omic data from TCGA and MET500, was used to corroborate transcriptional analysis and also provided information on transcriptional differences across tumor stages and on methylation profiles. Individual patient data from TCGA (Cell 2015 database) was downloaded through cBioPortal to analyze the correlation between CNAs and expression levels.

\subsection{Outcome Analyses}

To evaluate the relationship between the gene amplifications and patient clinical prognosis in terms of relapse-free survival (RFS), the publicly available Kaplan-Meier Plotter Online platform (http:/ / www.kmplot.com, accessed on: 8 January 2021) was used, as described previously [25]. Briefly, this tool is capable to assess the effect of $54 \mathrm{k}$ genes (mRNA, miRNA, proteins) on survival in multiple cancer types including BC $(n=6234)$, and using GEO, EGA, and TCGA as primary sources. Patients in the database were separated according to the median gene expression values. Patients above the threshold were labelled as "high" expressing ones, while patients below the threshold were labelled as "low" expressing. The two groups were compared using Cox survival analysis.

To analyze the correlation between mutations and patient clinical outcome, the publicly available Genotype-2-Outcome online platform (http:/ /www.g-2-o.com, accessed on: 8 January 2021) was interrogated, as described in previous studies [27]. Briefly, the database allows the association with prognosis of a specific transcriptomic signature linked to a mutation, by classifying patients according to the mean expression of the top 100 most related genes as a surrogate marker of its mutation status. Gene expression is compared between the mutational-carrying and the wild type population and those genes reaching significance are defined as the mutation signature. The median expression values for different transcripts are used as a cut-off to discriminate "high" and "low" expression cohorts, which are compared using a Cox survival analysis. For both tools, patients were stratified by their BC subtype using the PAM50 criteria.

\subsection{Co-Occurrence}

Co-occurrence analysis for gene alterations was evaluated using the cBioPortal online platform (www.cbioportal.org, accessed on: 8 January 2021). This tool calculates an odds ratio $(\mathrm{OR})$ for each pair of query genes, indicating the likelihood that the alterations for the two genes are co-occurrent in the selected cases, by the application of a Fisher's exact test (statistical significance $p<0.05$ ). 


\subsection{Construction and Analysis of PPI Networks and Functional Annotation}

We used the online tool STRING (http:/ / www.string-db.org) to construct interactome maps of amplified genes in all subtypes of BC. The closer the local clustering coefficient is to 1 , the more likely it is for the network to form clusters. PPI enrichment $p$-value indicates the statistical significance. Proteins are considered hubs when they have more interactions than the average.

\subsection{Search for Predictive Biomarkers}

To evaluate the relationship between gene amplifications and response to a specific therapy, the publicly-available ROC Plotter online tool (http:/ / www.rocplot.org/, accessed on: 8 January 2021) was used, as described previously [26]. Briefly, this tool is capable to link gene expression and response to therapy using transcriptomic data from 3104 breast tumor samples and using GEO, EGA, and TCGA as primary sources. The effect of each gene was interrogated in the (neo)adjuvant setting with endocrine therapy or chemotherapy across the different intrinsic subtypes. A box plot displaying expression levels in responders and non-responders ( $\mathrm{pCR}$ or 5 years RFS) is provided along with the area under the curve (AUC) and their respective $p$-values. Patients were stratified by their BC subtype using the PAM50 criteria.

\subsection{Functional Characterization of Mutations}

To evaluate the functional effects of the mutations found in the TCGA dataset, several publicly-available online predictor tools were consulted, including SIFT (https:/ / sift. bii.a-star.edu.sg/, accessed on: 8 January 2021; PolyPhen (http:/ / genetics.bwh.harvard. edu, accessed on: 8 January 2021), MutationTester (http:/ /www.mutationtaster.org/, accessed on: 8 January 2021), and FATHMM-MKL (http:/ / fathmm.biocompute.org.uk/, accessed on: 8 January 2021). MaxEntScan was used to estimate if mutations occurring in a splicing site impact the site function (http://hollywood.mit.edu/burgelab/ maxent/Xmaxentscan_scoreseq.html, accessed on: 8 January 2021).

\subsection{Analysis of Breast Cancer Cell Lines}

We interrogated the Cancer Dependency Map (DepMap) portal (https:/ / depmap.org/ portal/, accessed on: 8 January 2021), which comprises genomic profiles of hundreds of cancer cell line models and their sensitivity to genetic and small molecule perturbations, and also analyzed individual cell line mutational and transcriptomic data available at the Catalogue of Somatic Mutations in Cancer (COSMIC; https:/ / cancer.sanger.ac.uk/cell_ lines, accessed on: 8 January 2021) and at the Cancer Cell Line Encyclopedia (CCLE; https:/ / portals.broadinstitute.org/ccle, accessed on: 8 January 2021). The categorization of cell lines within each intrinsic subtype was performed according to the studies of Dai et al. and Kao et al. and transcriptional data [30,31]. The predictive capability of the set of gene amplifications was explored using data from up to four comprehensive online repositories including Cancer Target Discovery and Development (CTD), Genomics of Drug Sensitivity in Cancer (GDSC1 and GDSC2), and Profiling Relative Inhibition Simultaneously in Mixtures (PRISM), as available in the DepMap portal. Only those with data from at least 5 cell lines were selected for analytical purposes.

\subsection{Graphical Design}

Bars, heatmaps, and regression lines, were represented using GraphPad Prism software (version 8, GraphPad Software, San Diego, CA, USA) in terms of absolute counts, relative frequencies, and hazard ratios. Kaplan-Meier curves were produced by specific online tools as previously described (Kaplan-Meier plotter, Genotype2Outcome, ROC plotter). The interaction network was mapped as default by the STRING database analysis. 


\section{Conclusions}

In summary, we performed a comprehensive in silico study of the SUN machinery in breast tumors in search of potential targets for drug development and disease biomarkers. By using a wide repertoire of publicly available databases and analytical tools, we described three gene amplifications (UBE2T, UBE2C, BIRC5) with robust prognostic and predictive relevance in luminal and basal-like tumors, and two mutated genes (USP9X, USP7) whose surrogate transcriptional signatures have prognostic relevance in luminal A, HER2-enriched, and basal-like tumors.

Aware of the enormous potential, limitations, and biases intrinsic to computational approaches in cancer genomic research, we advocate for the design of analytical pipelines built on an utmost effort to reduce data noise and with the goal of providing early evidence of potential druggable targets and biomarkers for the future benefit of patients.

Supplementary Materials: The following are available online at https: / www.mdpi.com/2072-6 694/13/4/833/s1, Figure S1: Heatmap of all the genes retrieved in the ubiquitination, neddylation and SUMOylation processes with a frequency of amplification under $2 \%$, Figure S2: Transcriptomic, translational, and methylation correlates of amplified genes, Figure S3: Mutation-associated transcriptomic signatures, Figure S4: Analysis of the predictive capability of UBE2C and BIRC5 in luminal cell lines, Figure S5: Analysis of the predictive capability of UBE2T in TNBC cell lines, Table S1: Validation of the prognostic impact on survival in the TCGA and METABRIC datasets, Table S2: Validation of the impact of $U B E 2 T, U B E 2 C$, and BIRC5 on recurrence-free survival across intrinsic subtypes in the METABRIC and TCGA datasets, Table S3: Characterization of mutations identified in USP9X and USP7 in TCGA. Prediction of functional outcomes, Table S4: Characterization of a large panel of breast cancer cell lines in search of in vitro models for the identified genomic vulnerabilities.

Author Contributions: A.O. conceived the study and did the original design of analysis. J.F.-A., A.L.A.-S., E.C.M., V.G.-B., and M.d.M.N.-L. searched the data and performed the analyses. I.L-C., V.G.-B., and B.G. provided bioinformatic assistance in data search and interpretation. A.O. and J.F.-A. wrote the manuscript. All authors reviewed, included modification and approved the final version of the manuscript. All authors have read and agreed to the published version of the manuscript.

Funding: Work in Alberto Ocaña's lab is supported by the Instituto de Salud Carlos III (ISCIII, PI19/00808); ACEPAIN; Diputación de Albacete; and the CRIS Cancer Foundation. Work in Atanasio Pandiella's lab is supported by the Ministry of Economy and Competitiveness of Spain (BFU201571371-R, the Junta de Castilla y León (CSI146P20), and the CRIS Foundation. Balázs Györffy is financed by the 2018-2.1.17-TET-KR-00001 grant and by the Higher Education Institutional Excellence Programme of the Ministry for Innovation and Technology (MIT) in Hungary, within the framework of the Bionic thematic programme of the Semmelweis University.

Institutional Review Board Statement: Not applicable.

Informed Consent Statement: Not applicable.

Data Availability Statement: The data presented in this study are available within the article and its supplementary material.

Acknowledgments: We would like to thank our Funding Institutions, Instituto de Salud Carlos III, Diputación de Albacete, AECC Foundation, Ministry of Economy and Competitiveness of Spain, the Spanish Cancer Centers Network Program and the European Commission as the FEDER funding program responsible institution. We would like to specially thank our supporting foundations, as the "Asociación Costuras en la Piel en Apoyo a la investigación de cáncer en Albacete" (ACEPAIN) and the Cris Cancer Foundation for their continuous effort to support our work. The authors wish to acknowledge the support of ELIXIR Hungary (www.elixir-hungary.org).

Conflicts of Interest: The authors declare no conflict of interest.

\section{References}

1. Lord, S.J.; Kiely, B.E.; Pearson, S.-A.; Daniels, B.; O'Connell, D.L.; Beith, J.; Bulsara, M.K.; Houssami, N. Metastatic Breast Cancer Incidence, Site and Survival in Australia, 2001-2016: A Population-Based Health Record Linkage Study Protocol. BMJ Open 2019, 9. [CrossRef] [PubMed] 
2. International Cancer Genome Consortium; Hudson, T.J.; Anderson, W.; Artez, A.; Barker, A.D.; Bell, C.; Bernabé, R.R.; Bhan, M.K.; Calvo, F.; Eerola, I.; et al. International Network of Cancer Genome Projects. Nature 2010, 464, 993-998. [CrossRef] [PubMed]

3. Cancer Genome Atlas Network. Comprehensive Molecular Portraits of Human Breast Tumours. Nature 2012, 490, 61-70. [CrossRef] [PubMed]

4. Weinstein, J.N.; Collisson, E.A.; Mills, G.B.; Shaw, K.M.; Ozenberger, B.A.; Ellrott, K.; Shmulevich, I.; Sander, C.; Stuart, J.M. The Cancer Genome Atlas Pan-Cancer Analysis Project. Nat Genet. 2013, 45, 1113-1120. [CrossRef] [PubMed]

5. Pereira, B.; Chin, S.-F.; Rueda, O.M.; Vollan, H.-K.M.; Provenzano, E.; Bardwell, H.A.; Pugh, M.; Jones, L.; Russell, R.; Sammut, S.-J.; et al. The Somatic Mutation Profiles of 2433 Breast Cancers Refine Their Genomic and Transcriptomic Landscapes. Nat. Commun. 2016, 7, 11479. [CrossRef]

6. Razavi, P.; Chang, M.T.; Xu, G.; Bandlamudi, C.; Ross, D.S.; Vasan, N.; Cai, Y.; Bielski, C.M.; Donoghue, M.T.A.; Jonsson, P.; et al. The Genomic Landscape of Endocrine-Resistant Advanced Breast Cancers. Cancer Cell 2018, 34, 427-438. [CrossRef]

7. Perou, C.M.; Sørlie, T.; Eisen, M.B.; van de Rijn, M.; Jeffrey, S.S.; Rees, C.A.; Pollack, J.R.; Ross, D.T.; Johnsen, H.; Akslen, L.A.; et al. Molecular Portraits of Human Breast Tumours. Nature 2000, 406, 747-752. [CrossRef]

8. Koboldt, D.C.; Fulton, R.S.; McLellan, M.D.; Schmidt, H.; Kalicki-Veizer, J.; McMichael, J.F.; Fulton, L.L.; Dooling, D.J.; Ding, L.; Mardis, E.R.; et al. Comprehensive Molecular Portraits of Human Breast Tumours. Nature 2012, 490, 61-70. [CrossRef]

9. Condorelli, R.; Mosele, F.; Verret, B.; Bachelot, T.; Bedard, P.L.; Cortes, J.; Hyman, D.M.; Juric, D.; Krop, I.; Bieche, I.; et al. Genomic Alterations in Breast Cancer: Level of Evidence for Actionability According to ESMO Scale for Clinical Actionability of Molecular Targets (ESCAT). Ann. Oncol. 2019, 30, 365-373. [CrossRef]

10. Krueger, K.E.; Srivastava, S. Posttranslational Protein Modifications: Current Implications for Cancer Detection, Prevention, and Therapeutics. Mol. Cell. Proteomics 2006, 5, 1799-1810. [CrossRef]

11. Gâtel, P.; Piechaczyk, M.; Bossis, G. Ubiquitin, SUMO, and Nedd8 as Therapeutic Targets in Cancer. Adv. Exp. Med. Biol. 2020, 1233, 29-54. [CrossRef]

12. Scheffner, M.; Nuber, U.; Huibregtse, J.M. Protein Ubiquitination Involving an E1-E2-E3 Enzyme Ubiquitin Thioester Cascade. Nature 1995, 373, 81-83. [CrossRef] [PubMed]

13. Gallo, L.H.; Ko, J.; Donoghue, D.J. The Importance of Regulatory Ubiquitination in Cancer and Metastasis. Cell Cycle 2017, 16, 634-648. [CrossRef]

14. Bologna, S.; Ferrari, S. It Takes Two to Tango: Ubiquitin and SUMO in the DNA Damage Response. Front Genet 2013, 4, 106. [CrossRef] [PubMed]

15. Naik, S.K.; Lam, E.W.-F.; Parija, M.; Prakash, S.; Jiramongkol, Y.; Adhya, A.K.; Parida, D.K.; Mishra, S.K. NEDDylation Negatively Regulates ERR $\beta$ Expression to Promote Breast Cancer Tumorigenesis and Progression. Cell Death Dis. 2020, 11, 703. [CrossRef]

16. Hungria, V.T. de M.; Crusoé, E. de Q.; Bittencourt, R.I.; Maiolino, A.; Magalhães, R.J.P.; Sobrinho, J. do N.; Pinto, J.V.; Fortes, R.C.; Moreira, E. de S.; Tanaka, P.Y. New Proteasome Inhibitors in the Treatment of Multiple Myeloma. Hematol. Transfus. Cell Ther. 2019, 41, 76-83. [CrossRef]

17. Wang, W.; Qin, J.-J.; Voruganti, S.; Srivenugopal, K.S.; Nag, S.; Patil, S.; Sharma, H.; Wang, M.-H.; Wang, H.; Buolamwini, J.K.; et al. The Pyrido[b]Indole MDM2 Inhibitor SP-141 Exerts Potent Therapeutic Effects in Breast Cancer Models. Nat. Commun. 2014, 5, 5086. [CrossRef] [PubMed]

18. Lemos, A.; Leão, M.; Soares, J.; Palmeira, A.; Pinto, M.; Saraiva, L.; Sousa, M.E. Medicinal Chemistry Strategies to Disrupt the P53-MDM2/MDMX Interaction. Med. Res. Rev. 2016, 36, 789-844. [CrossRef]

19. Arvinas Receives Authorization to Proceed for ARV-471, a PROTAC@Protein Degrader to Treat Patients with Locally Advanced or Metastatic ER+/HER2- Breast Cancer. Available online: https:/ /ir.arvinas.com/news-releases/news-release-details/arvinasreceives-authorization-proceed-arv-471-protacr-protein/ (accessed on 3 December 2020).

20. Cerami, E.; Gao, J.; Dogrusoz, U.; Gross, B.E.; Sumer, S.O.; Aksoy, B.A.; Jacobsen, A.; Byrne, C.J.; Heuer, M.L.; Larsson, E.; et al. The CBio Cancer Genomics Portal: An Open Platform for Exploring Multidimensional Cancer Genomics Data. Cancer Discov. 2012, 2, 401-404. [CrossRef]

21. Gao, J.; Aksoy, B.A.; Dogrusoz, U.; Dresdner, G.; Gross, B.; Sumer, S.O.; Sun, Y.; Jacobsen, A.; Sinha, R.; Larsson, E.; et al. Integrative Analysis of Complex Cancer Genomics and Clinical Profiles Using the CBioPortal. Sci. Signal. 2013, 6, 11. [CrossRef] [PubMed]

22. Tang, Z.; Kang, B.; Li, C.; Chen, T.; Zhang, Z. GEPIA2: An Enhanced Web Server for Large-Scale Expression Profiling and Interactive Analysis. Nucleic Acids Res. 2019, 47, W556-W560. [CrossRef]

23. Chandrashekar, D.S.; Bashel, B.; Balasubramanya, S.A.H.; Creighton, C.J.; Ponce-Rodriguez, I.; Chakravarthi, B.V.S.K.; Varambally, S. UALCAN: A Portal for Facilitating Tumor Subgroup Gene Expression and Survival Analyses. Neoplasia 2017, 19, 649-658. [CrossRef]

24. Jézéquel, P.; Frénel, J.-S.; Campion, L.; Guérin-Charbonnel, C.; Gouraud, W.; Ricolleau, G.; Campone, M. Bc-GenExMiner 3.0: New Mining Module Computes Breast Cancer Gene Expression Correlation Analyses. Database (Oxford) 2013, 2013. [CrossRef]

25. Györffy, B.; Lanczky, A.; Eklund, A.C.; Denkert, C.; Budczies, J.; Li, Q.; Szallasi, Z. An Online Survival Analysis Tool to Rapidly Assess the Effect of 22,277 Genes on Breast Cancer Prognosis Using Microarray Data of 1,809 Patients. Breast Cancer Res. Treat. 2010, 123, 725-731. [CrossRef] [PubMed]

26. Fekete, J.T.; Győrffy, B. ROCplot.Org: Validating Predictive Biomarkers of Chemotherapy/Hormonal Therapy/Anti-HER2 Therapy Using Transcriptomic Data of 3,104 Breast Cancer Patients. Int. J. Cancer 2019, 145, 3140-3151. [CrossRef] 
27. Pongor, L.; Kormos, M.; Hatzis, C.; Pusztai, L.; Szabó, A.; Győrffy, B. A Genome-Wide Approach to Link Genotype to Clinical Outcome by Utilizing next Generation Sequencing and Gene Chip Data of 6,697 Breast Cancer Patients. Genome Med. 2015, 7, 104. [CrossRef]

28. Tate, J.G.; Bamford, S.; Jubb, H.C.; Sondka, Z.; Beare, D.M.; Bindal, N.; Boutselakis, H.; Cole, C.G.; Creatore, C.; Dawson, E.; et al. COSMIC: The Catalogue Of Somatic Mutations In Cancer. Nucleic Acids Res. 2019, 47, D941-D947. [CrossRef] [PubMed]

29. Barretina, J.; Caponigro, G.; Stransky, N.; Venkatesan, K.; Margolin, A.A.; Kim, S.; Wilson, C.J.; Lehár, J.; Kryukov, G.V.; Sonkin, D.; et al. The Cancer Cell Line Encyclopedia Enables Predictive Modelling of Anticancer Drug Sensitivity. Nature 2012, 483, 603-607. [CrossRef] [PubMed]

30. Dai, X.; Cheng, H.; Bai, Z.; Li, J. Breast Cancer Cell Line Classification and Its Relevance with Breast Tumor Subtyping. J. Cancer 2017, 8, 3131-3141. [CrossRef]

31. Kao, J.; Salari, K.; Bocanegra, M.; Choi, Y.-L.; Girard, L.; Gandhi, J.; Kwei, K.A.; Hernandez-Boussard, T.; Wang, P.; Gazdar, A.F.; et al. Molecular Profiling of Breast Cancer Cell Lines Defines Relevant Tumor Models and Provides a Resource for Cancer Gene Discovery. PLOS ONE 2009, 4, e6146. [CrossRef]

32. Dastsooz, H.; Cereda, M.; Donna, D.; Oliviero, S. A Comprehensive Bioinformatics Analysis of UBE2C in Cancers. Int. J. Mol. Sci. 2019, 20, 2228. [CrossRef]

33. Psyrri, A.; Kalogeras, K.T.; Kronenwett, R.; Wirtz, R.M.; Batistatou, A.; Bournakis, E.; Timotheadou, E.; Gogas, H.; Aravantinos, G.; Christodoulou, C.; et al. Prognostic Significance of UBE2C MRNA Expression in High-Risk Early Breast Cancer. A Hellenic Cooperative Oncology Group (HeCOG) Study. Ann. Oncol. 2012, 23, 1422-1427. [CrossRef] [PubMed]

34. Loussouarn, D.; Campion, L.; Leclair, F.; Campone, M.; Charbonnel, C.; Ricolleau, G.; Gouraud, W.; Bataille, R.; Jézéquel, P. Validation of UBE2C Protein as a Prognostic Marker in Node-Positive Breast Cancer. Br. J. Cancer 2009, 101, 166-173. [CrossRef]

35. Mo, C.; Gao, L.; Zhu, X.; Wei, K.; Zeng, J.; Chen, G.; Feng, Z. The Clinicopathological Significance of UBE2C in Breast Cancer: A Study Based on Immunohistochemistry, Microarray and RNA-Sequencing Data. Cancer Cell International 2017, 17, 83. [CrossRef]

36. Perez-Peña, J.; Corrales-Sánchez, V.; Amir, E.; Pandiella, A.; Ocana, A. Ubiquitin-Conjugating Enzyme E2T (UBE2T) and Denticleless Protein Homolog (DTL) Are Linked to Poor Outcome in Breast and Lung Cancers. Sci. Rep. 2017, 7. [CrossRef] [PubMed]

37. Kim, Y.-J.; Lee, G.; Han, J.; Song, K.; Choi, J.-S.; Choi, Y.-L.; Shin, Y.K. UBE2C Overexpression Aggravates Patient Outcome by Promoting Estrogen-Dependent/Independent Cell Proliferation in Early Hormone Receptor-Positive and HER2-Negative Breast Cancer. Front. Oncol. 2020, 9. [CrossRef] [PubMed]

38. Rawat, A.; Gopal, G.; Selvaluxmy, G.; Rajkumar, T. Inhibition of Ubiquitin Conjugating Enzyme UBE2C Reduces Proliferation and Sensitizes Breast Cancer Cells to Radiation, Doxorubicin, Tamoxifen and Letrozole. Cell. Oncol. (Dordr) 2013, 36, 459-467. [CrossRef]

39. Ueki, T.; Park, J.-H.; Nishidate, T.; Kijima, K.; Hirata, K.; Nakamura, Y.; Katagiri, T. Ubiquitination and Downregulation of BRCA1 by Ubiquitin-Conjugating Enzyme E2T Overexpression in Human Breast Cancer Cells. Cancer Res. 2009, 69, 8752-8760. [CrossRef] [PubMed]

40. Dai, J.; Zhu, B.; Lin, W.; Gao, H.; Dai, H.; Zheng, L.; Shi, W.; Chen, W. Identification of Prognostic Significance of BIRC5 in Breast Cancer Using Integrative Bioinformatics Analysis. Biosci. Rep. 2020, 40. [CrossRef]

41. Hamy, A.S.; Bieche, I.; Lehmann-Che, J.; Scott, V.; Bertheau, P.; Guinebretière, J.M.; Matthieu, M.C.; Sigal-Zafrani, B.; Tembo, O.; Marty, M.; et al. BIRC5 (Survivin): A Pejorative Prognostic Marker in Stage II/III Breast Cancer with No Response to Neoadjuvant Chemotherapy. Breast Cancer Res. Treat. 2016, 159, 499-511. [CrossRef]

42. Lyu, H.; Huang, J.; He, Z.; Liu, B. Epigenetic Mechanism of Survivin Dysregulation in Human Cancer. Sci. China Life Sci. 2018, 61, 808-814. [CrossRef]

43. Nabilsi, N.H.; Broaddus, R.R.; Loose, D.S. DNA Methylation Inhibits P53-Mediated Survivin Repression. Oncogene 2009, 28, 2046-2050. [CrossRef] [PubMed]

44. Wang, C.; Zheng, X.; Shen, C.; Shi, Y. MicroRNA-203 Suppresses Cell Proliferation and Migration by Targeting BIRC5 and LASP1 in Human Triple-Negative Breast Cancer Cells. J. Exp. Clin. Cancer Res. 2012, 31, 58. [CrossRef]

45. Véquaud, E.; Desplanques, G.; Jézéquel, P.; Juin, P.; Barillé-Nion, S. Survivin Contributes to DNA Repair by Homologous Recombination in Breast Cancer Cells. Breast Cancer Res. Treat. 2016, 155, 53-63. [CrossRef] [PubMed]

46. Lu, Q.; Zhang, F.-L.; Lu, D.-Y.; Shao, Z.-M.; Li, D.-Q. USP9X Stabilizes BRCA1 and Confers Resistance to DNA-Damaging Agents in Human Cancer Cells. Cancer Med. 2019, 8, 6730-6740. [CrossRef]

47. Oosterkamp, H.M.; Hijmans, E.M.; Brummelkamp, T.R.; Canisius, S.; Wessels, L.F.A.; Zwart, W.; Bernards, R. USP9X Downregulation Renders Breast Cancer Cells Resistant to Tamoxifen. Cancer Res. 2014, 74, 3810-3820. [CrossRef] [PubMed]

48. Li, X.; Song, N.; Liu, L.; Liu, X.; Ding, X.; Song, X.; Yang, S.; Shan, L.; Zhou, X.; Su, D.; et al. USP9X Regulates Centrosome Duplication and Promotes Breast Carcinogenesis. Nat. Commun. 2017, 8, 14866. [CrossRef]

49. Li, L.; Liu, T.; Li, Y.; Wu, C.; Luo, K.; Yin, Y.; Chen, Y.; Nowsheen, S.; Wu, J.; Lou, Z.; et al. The Deubiquitinase USP9X Promotes Tumor Cell Survival and Confers Chemoresistance through YAP1 Stabilization. Oncogene 2018, 37, 2422-2431. [CrossRef]

50. Giovinazzi, S.; Morozov, V.M.; Summers, M.K.; Reinhold, W.C.; Ishov, A.M. USP7 and Daxx Regulate Mitosis Progression and Taxane Sensitivity by Affecting Stability of Aurora-A Kinase. Cell Death Differ. 2013, 20, 721-731. [CrossRef] 
51. Hernández-Pérez, S.; Cabrera, E.; Salido, E.; Lim, M.; Reid, L.; Lakhani, S.R.; Khanna, K.K.; Saunus, J.M.; Freire, R. DUB3 and USP7 De-Ubiquitinating Enzymes Control Replication Inhibitor Geminin: Molecular Characterization and Associations with Breast Cancer. Oncogene 2017, 36, 4802-4809. [CrossRef] [PubMed]

52. Xia, X.; Liao, Y.; Huang, C.; Liu, Y.; He, J.; Shao, Z.; Jiang, L.; Dou, Q.P.; Liu, J.; Huang, H. Deubiquitination and Stabilization of Estrogen Receptor $\alpha$ by Ubiquitin-Specific Protease 7 Promotes Breast Tumorigenesis. Cancer Letters 2019, 465, 118-128. [CrossRef] [PubMed]

53. Cappadocia, L.; Lima, C.D. Ubiquitin-like Protein Conjugation: Structures, Chemistry, and Mechanism. Chem. Rev. 2018, 118, 889-918. [CrossRef] [PubMed]

54. Li, F.; Aljahdali, I.; Ling, X. Cancer Therapeutics Using Survivin BIRC5 as a Target: What Can We Do after over Two Decades of Study? J. Exp. Clin. Cancer Res. 2019, 38, 368. [CrossRef] [PubMed]

55. Deng, L.; Meng, T.; Chen, L.; Wei, W.; Wang, P. The Role of Ubiquitination in Tumorigenesis and Targeted Drug Discovery. Signal Transduct. Target. Ther. 2020, 5, 1-28. [CrossRef]

56. Science-Mission Therapeutics. Available online: https://missiontherapeutics.com/science/ (accessed on 8 January 2021). 